\title{
Hyperactivity of Newborn Pten Knock-out Neurons Results from Increased Excitatory Synaptic Drive
}

\author{
Michael R. Williams, Tyrone DeSpenza Jr, Meijie Li, Allan T. Gulledge, and Bㅏㅁan W. Luikart \\ Department of Physiology and Neurobiology, Geisel School of Medicine at Dartmouth College, Lebanon, New Hampshire 03756
}

\begin{abstract}
Developing neurons must regulate morphology, intrinsic excitability, and synaptogenesis to form neural circuits. When these processes go awry, disorders, including autism spectrum disorder (ASD) or epilepsy, may result. The phosphatase Pten is mutated in some patients having ASD and seizures, suggesting that its mutation disrupts neurological function in part through increasing neuronal activity. Supporting this idea, neuronal knock-out of Pten in mice can cause macrocephaly, behavioral changes similar to ASD, and seizures. However, the mechanisms through which excitability is enhanced following Pten depletion are unclear. Previous studies have separately shown that Pten-depleted neurons can drive seizures, receive elevated excitatory synaptic input, and have abnormal dendrites. We therefore tested the hypothesis that developing Pten-depleted neurons are hyperactive due to increased excitatory synaptogenesis using electrophysiology, calcium imaging, morphological analyses, and modeling. This was accomplished by coinjecting retroviruses to either "birthdate" or birthdate and knock-out Pten in granule neurons of the murine neonatal dentate gyrus. We found that Pten knock-out neurons, despite a rapid onset of hypertrophy, were more active in vivo. Pten knock-out neurons fired at more hyperpolarized membrane potentials, displayed greater peak spike rates, and were more sensitive to depolarizing synaptic input. The increased sensitivity of Pten knock-out neurons was due, in part, to a higher density of synapses located more proximal to the soma. We determined that increased synaptic drive was sufficient to drive hypertrophic Pten knock-out neurons beyond their altered action potential threshold. Thus, our work contributes a developmental mechanism for the increased activity of Pten-depleted neurons.
\end{abstract}

Key words: autism spectrum disorder; dendritic spine; neuron structure function; Pten; seizure; synaptogenesis

\section{Introduction}

The biology underlying the proper formation and function of neural circuits is of central interest in neuroscience. Genetic mutations found in patients with neurodevelopmental disorders give experimental inroads to, and highlight the clinical importance of, understanding these processes. Mutations in the dualspecificity lipid and protein phosphatase, Phosphatase and Tensin Homolog on Chromosome Ten (PTEN), exist in some patients having autism spectrum disorder (ASD) with macrocephaly (Goffin et al., 2001; Butler et al., 2005; Buxbaum et al., 2007; Orrico et al., 2009; Varga et al., 2009; McBride et al., 2010; Stein et al., 2010; Conti et al., 2012; Klein et al., 2013; Hobert et al., 2014). Pten normally functions to oppose the PI3K-Akt-mTor pathway by catalyzing the reverse reaction of PI3K: degrading

Received July 30, 2014; revised Nov. 12, 2014; accepted Nov. 19, 2014.

Author contributions: M.R.W., A.T.G., and B.W.L. designed research; M.R.W., T.D., M.L., A.T.G., and B.W.L. performed research; M.R.W., T.D., A.T.G., and B.W.L. analyzed data; M.R.W., A.T.G., and B.W.L. wrote the paper.

This work was supported by National Institutes of Health Grant 5 R01 MH097949 to B.W.L. and Grant 1 R01 MH099054 to A.T.G., Autism Speaks Pilot Grant 7359 to B.W.L., National Science Foundation Grant MRI 0922631 to A.T.G., and the Optical Cellular Imaging Shared Resource and the Norris Cotton Cancer Center at the Geisel School of Medicine at Dartmouth (5 P30 CA023108). We thank Jeonghoon Lee, Derek R. Racine, Grace B. Russo, and Paul W. Frazel for their contributions to data acquisition and analysis.

The authors declare no competing financial interests.

Correspondence should be addressed to Dr. Bryan W. Luikart, Geisel School of Medicine at Dartmouth, Physiology and Neurobiology, 1 Medical Center Drive, Borwell 708E, Lebanon, NH 03745. E-mail: Bryan.W.Luikart@Dartmouth.edu.

DOI:10.1523/JNEUROSCI.3144-14.2015

Copyright $\odot 2015$ the authors $\quad 0270-6474 / 15 / 350943-17 \$ 15.00 / 0$ phosphatidylinositol 3,4,5-trisphosphate to phosphatidylinositol (4,5)-biphosphate. Further, Pten likely has important scaffolding and protein-phosphatase roles in regulating neuronal cell biology. Through mechanisms not yet elucidated, ASD-associated point mutations are thought to lead to protein dysfunction in one or more of these cellular roles, ultimately causing pathological changes in neurophysiology.

Seizures and ASD are present in $\sim 30 \%$ of patients with either disorder (Tuchman et al., 2010), and PTEN mutations are documented in patients with epilepsy and ASD (Rudolph et al., 2011; Marchese et al., 2014). This suggests that loss of Pten protein function is sufficient to increase neuronal activity to pathological levels and that this increased neuronal activity may be etiological in the associated forms of ASD or epilepsy. Supporting this idea, Cre/Lox strategies in mice have been used to uncover how loss of Pten produces changes in neuronal form and function, but the varied developmental stages at which Pten has been depleted, and the variable times over which phenotypes have been studied, has produced seemingly conflicting data and interpretations regarding the relationship between morphological changes and physiology (Backman et al., 2001; Kwon et al., 2001, 2006; Ogawa et al., 2007; Zhou et al., 2009; Amiri et al., 2012; Sperow et al., 2012; Lugo et al., 2014). Consequently, the mechanisms connecting the loss of function of Pten to altered neuronal excitability have remained unresolved. Interestingly, it has been found that, when Pten is depleted from developing neurons, this results in increased excitatory afferent input (Luikart et al., 2011a; Xiong et 
al., 2012) and can lead to seizures (Amiri et al., 2012; Pun et al., 2012). Thus, we tested the hypothesis that loss of Pten function increases neuronal excitability through increased developmental excitatory synaptogenesis.

We coinjected retroviruses that encoded a fluorescent protein only with retroviruses that encoded a distinct fluorescent protein and Cre into the dentate gyrus of neonatal Pten-floxed mice. This allowed us to birthdate, label, and manipulate Pten in newborn granule neurons. By comparing Pten knock-out (KO) neurons to control (control) neurons of the same age within the same animal, we measured cell-autonomous changes caused by Pten KO. We found that increased developmental dendrite arborization and recruitment of excess excitatory inputs explain the increased synaptic drive underlying the excessive activity of Pten KO neurons.

\section{Materials and Methods}

Animals. Procedures were approved by the Dartmouth Institutional Animal Care and Use Committee and conformed to federal, state, local, and Association for Assessment and Accreditation of Laboratory Animal Care standards. The animals used in these experiments were mice of either gender, supplied by The Jackson Laboratory. The two strains utilized were C57BL/6J (control) and B6.129S4-Pten $<$ tmlhwu $>/$ J homozygous ("Pten Floxed") mice, which were also on the C57BL/6J genetic background, of either gender (Kwon et al., 2006; Luikart et al., 2008). Animals were on a $12 \mathrm{~h}$ light/dark cycle with chow and water provided ad libitum.

Surgery and slice preparation. Under isoflurane anesthesia, $2 \mu \mathrm{l}$ bilateral injections of replication-defective retroviruses based on pRubi were made into the dentate gyrus at postnatal day 7 , at a rate of at $0.3 \mu \mathrm{l} / \mathrm{min}$ (Bayer and Altman, 1974; Luikart et al., 2011b). At varying days postinjection (DPI), animals were anesthetized by intraperitoneal injection of $2 \%$ tribromoethanol and were transcardially perfused for immunohistochemistry or for electrophysiology and multiphoton microscopy. Slices were generated at $7.5,12.5,16.5,20.5$, and $24.5 \mathrm{DPI}( \pm 1 \mathrm{~d}$ at each DPI) using published methods (Luikart et al., 2011a).

Replication-defective retroviruses. Methods for production of replicationdefective retroviruses based on pRubi have been previously published (Luikart et al., 2011b); briefly, viral particles expressed either a fluorescent reporter alone or both a fluorescent reporter and Cre recombinase via a T2A motif (Donnelly et al., 2001).

To generate pRubi-mCherry-T2A-Cre, we used PCR to amplify and add a 5' BsrG1 and a 3' EcoR1 site to Cre recombinase. The PCR product was then ligated into a cloning plasmid. Using annealed oligos with BsrG1-compatible overhangs and BsrG1 digestion, we introduced a T2A element upstream of Cre. The T2A-Cre was excised from the cloning plasmid and introduced into FUGW or FUCW via EcoR1 and BsrG1 digestion to create FUeGFP-T2A-Cre and FUmCherry-T2A-Cre, respectively. To generate retroviral constructs, SacII sites were used to excise a fragment encoding the ubiquitin promoter, fluorescent protein-T2ACre, and WRE elements from lentivirus plasmids, which were ligated into pRubi that had also been SacII digested.

To generate a retrovirus expressing the ultrasensitive calcium indicator GCaMP6s, we procured pGP-CMV-GCaMP6s (Addgene: pGPCMV-GCaMP6s, Douglas Kim) (Chen et al., 2013), from which we amplified GCaMP6s, adding EcoR1 sites at each end by PCR. The amplification product was purified and ligated into a cloning plasmid. From pRubi, eGFP was excised by Xbal. Then, GCaMP6s was ligated into (pRubi minus eGFP) after both had been EcoR1 digested. All plasmids were confirmed by sequencing.

Immunohistochemistry. Methods for eGFP, mCherry, Pten, and relative phosphorylated ribosomal S6 (p-S6) immunolabeling, and methods for neuronal soma size analysis, have been previously published (Fricano et al., 2014). For c-Fos measurements, rabbit polyclonal anti-c-Fos (Oncogene Science/Nuclea Diagnostics) was used; intensity measurement normalization was done as for p-S6. Antibodies against synaptobrevin 2 $(1: 1000,104-211)$ and against Shank2 (1:400, 162-202) were from Syn- aptic Systems and were used on tissue that had undergone $\mathrm{pH} 6.0$ sodium citrate heat-mediated antigen retrieval. Reconstructions were accomplished by manual tracing in Neurolucida (MBF Bioscience).

Electrophysiology. The electrophysiological methods used here have been previously published (Luikart et al., 2011a; Rudolph et al., 2011). Tribromoethanol-anesthetized mice were perfused and hippocampal slices made in ice-cold solution containing the following (in $\mathrm{mm}$ ): 110 choline-Cl, 10 D-glucose, $7 \mathrm{MgCl}_{2}, 2.5 \mathrm{KCl}, 1.25 \mathrm{NaH}_{2} \mathrm{PO}_{4} \cdot 2 \mathrm{H}_{2} \mathrm{O}, 0.5$ $\mathrm{CaCl}_{2}, 1.3 \mathrm{Na}$-ascorbate, and $25 \mathrm{NaHCO}_{3}$, bubbled with $95 \% \mathrm{O}_{2}-5 \%$ $\mathrm{CO}_{2}$. Slices were stored at room temperature, and recordings were performed at $37^{\circ} \mathrm{C}$ in aCSF as follows (in mM): $125 \mathrm{NaCl}, 25 \mathrm{NaHCO}_{3}, 2.5$ $\mathrm{KCl}, 1.25 \mathrm{NaH}_{2} \mathrm{PO}_{4}, 2.0 \mathrm{CaCl}_{2}, 1.0 \mathrm{MgCl}_{2}$, and $25 \mathrm{D}$-glucose, bubbled with $95 \% \mathrm{O}_{2}-5 \% \mathrm{CO}_{2}$. Intrinsic properties and current-clamp recordings were performed using a K-gluconate internal solution as follows (in mM): 115 K-gluconate, 10 HEPES, 2 EGTA, $20 \mathrm{KCl}, 2 \mathrm{MgATP}, 10 \mathrm{Na}$ phosphocreatine, and $0.3 \mathrm{Na}_{3} \mathrm{GTP}$. Synaptic currents were recorded using Cs-gluconate internal solution containing the following (in $\mathrm{mM}$ ): 113 Cs gluconate, 10 HEPES, 10 EGTA, $17.5 \mathrm{CsCl}, 8 \mathrm{NaCl}, 2 \mathrm{MgATP}, 0.3$ NaGTP, 10 Na-phosphocreatine, pH 7.3, 295 mOsm. Recordings in strontium-containing extracellular solution replaced Ca using $2 \mathrm{~mm} \mathrm{Mg}$ and $3 \mathrm{~mm}$ Sr. Series resistance was monitored before and after recordings; data were discarded if it increased by $>10 \mathrm{M} \Omega$. Recordings were sampled at $80 \mathrm{kHz}$ (Multiclamp 700B; Molecular Devices). Action potentials were detected using a template (Axograph $\mathrm{X}$ ) and reviewed manually. Spike threshold was defined as the membrane potential at which the first derivative of the voltage trace reached $10 \%$ of its peak. Analysis of spike amplitude, rise time, and half-width was performed on action potentials evoked by $500 \mathrm{~ms}$ current injection, which were temporally isolated by at least $20 \mathrm{~ms}$ from any other spikes in the train. For determination of the afterhyperpolarization, only the first spike was analyzed, and the temporal window was $20 \mathrm{~ms}$. All other spike characteristics were measured by Axograph X. Asynchronous EPSCs (aEPSCs) were detected using template matching in Axograph X. The template rise time was $1 \mathrm{~ms}$, decay time $6 \mathrm{~ms}$, baseline $10 \mathrm{~ms}$, length $30 \mathrm{~ms}$, and had a threshold of 3 . All captured events were then manually reviewed. The miniature EPSC (mEPSC) recordings were made in the presence of $1 \mu \mathrm{M}$ TTX and $10 \mu \mathrm{M}$ SR95531 (Gabazine), using the potassium gluconate internal solution and a holding current of $-90 \mathrm{mV}$. For processing, mEPSC events were filtered at $2 \mathrm{~Hz}$ and the template kinetics were the same as used for aEPSCs.

Multiphoton microscopy. Morphology of granule neuron dendritic protrusions/spines in the territory of the outer molecular layer was captured by multiphoton microscopy (Ultima In Vivo, Prairie Technologies/Bruker). Slices were superfused with aCSF bubbled with $95 \% \mathrm{O}_{2}, 5 \%$ $\mathrm{CO}_{2}$, at $37^{\circ} \mathrm{C}$. Stimulation of fluorescence was at $920 \mathrm{~nm}$ using a Chameleon tunable laser (Coherent). Control neurons were those expressing only eGFP in non-Pten-floxed mice of the same genetic background (C57BL/6J, "wild-type", control) or Pten-floxed mice; Pten KO neurons were those expressing eGFP-T2A-Cre in Pten-floxed mice.

Morphology measurements. Image series were analyzed using ImageJ. After performing the "Smooth" function, a maximum intensity Z-projection was generated for each time point using the Group Z Projector plug-in. Small degrees of drift were corrected by the StackReg plug-in using the "Rigid Body" option.

A segmented line ROI was used to define dendrite length. ROI width was increased to encompass the dendrite shaft. From the area of the dendrite shaft ROI, dendrite width was calculated. For each apparent protrusion/spine, coordinates at the base of the protrusion were recorded. The number of protrusions divided by the length of the dendritic segment gave apparent protrusion density. For each protrusion, the Manual Tracking plug-in was used to plot coordinates of the spine tip at each time point. The length of each protrusion was approximated as the mean shortest distance between the coordinates of the protrusion base and the protrusion tip across each of the 10 time points of the imaging epoch.

Dendritic protrusions were classified by reviewers blinded to DPI and Pten status. Reviewers indicated type: thin/filopodial (longer than wide with no obvious head), stubby (wider than long with no obvious head), mushroom (discernibly wider head and thinner neck), or atypical (if it could not be clearly categorized as another morphological type). It was 
noted when a protrusion acquired a mushroom morphology over the imaging epoch. For these metrics, the count per dendritic segment was divided by the length of that dendritic segment to calculate the density. Densities were averaged between reviewers and used for final analyses.

Modeling. Morphologies for control and Pten KO neurons were reconstructed into multicompartment models in Neurolucida and imported as multicompartment models into NEURON (version 7.3). Axial resistivity and specific membrane capacitance were set to $100 \Omega \mathrm{cm}$ and $1 \mu \mathrm{F} / \mathrm{cm}^{2}$, respectively. Increased surface areas in dendrites generated by protrusions were calculated from measurements for protrusion densities, protrusion lengths, and dendritic diameters observed in control and Pten KO neurons (Tables 3 and 4; data from 20.5 to $24.5 \mathrm{DPI}$ ) with protrusions assumed to have a mean diameter of $0.25 \mu \mathrm{m}$ and with protrusion densities observed in Z-projections expected to detect 33\% of the total protrusions along dendrites. Specific membrane capacitance and specific membrane conductance of dendritic compartments was then multiplied by the resulting "spine factor" ( 1.54 for control, 1.89 for Pten KO), and specific membrane resistivity was adjusted to replicate the experimentally determined mean $\mathrm{R}_{\mathrm{N}}$ s for 20.5 and 24.5 DPI control $\left(\mathrm{R}_{\mathrm{M}}=17,850\right.$ $\Omega \mathrm{cm}^{2}$; mean $\left.\mathrm{R}_{\mathrm{N}}=555 \mathrm{M} \Omega\right)$ and Pten $\mathrm{KO}\left(\mathrm{R}_{\mathrm{M}}=13,160 \Omega \mathrm{cm}^{2}\right.$; mean $\mathrm{R}_{\mathrm{N}}=143 \mathrm{M} \Omega$ ) neurons. The leak conductance was set to reverse at -93 $\mathrm{mV}$. Synaptic inputs were simulated as AMPA-like conductance changes having exponential rise ( $\mathrm{tau}=0.2 \mathrm{~ms}$ ) and decay ( $\mathrm{tau}=2 \mathrm{~ms}$ ), a maximum conductance of $350 \mathrm{pS}$ (chosen to match mean aEPSC amplitudes in control neurons), and reversal potentials of $0 \mathrm{mV}$. Synapses were individually activated at $1 \mu \mathrm{m}$ intervals along all dendritic segments, and responses were recorded with a simulated somatic single-electrode voltage clamp. All simulated synaptic responses in a given neuron were averaged to generate a mean EPSC, from which the population mean EPSC ( \pm 2 SEM), was generated (see Fig. 9).

Statistics. Toward ensuring that our studies had adequate statistical power, sample size was guided by our previously published research that used similar immunohistochemical and electrophysiological techniques (Luikart et al., 2011a; Fricano et al., 2014). Where absence of a statistically significant difference was biologically relevant to data interpretation, we explicitly analyzed power post hoc test using GraphPad StatMate 2.0. To compare Pten $\mathrm{KO}$ versus control neurons across time points, two-way ANOVA with Bonferroni's multiple-comparisons test was used. For electrophysiological studies comparing control versus Pten KO neuron responses to increasing levels of afferent stimulation or for immunohistochemical studies comparing control versus Pten KO neurons within the same animal, two-way repeated-measures ANOVA with Bonferroni's multiple-comparison test was used. Where control versus Pten $\mathrm{KO}$ neurons were compared at a single time point, Student's $t$ tests were used with Welch's correction where appropriate.

\section{Results}

\section{Retroviral Pten deletion causes hypertrophy and increases markers of activity in developing neurons}

To investigate the development of Pten KO neurons, we generated retroviruses in which Cre was downstream of a fluorescent protein via a T2A motif (Donnelly et al., 2001). We then coinjected retroviruses encoding only a fluorescent protein with retroviruses encoding a distinct fluorescent protein-T2A-Cre, into the dentate gyrus of P7 Pten flx/flx animals (Lesche et al., 2002) (Fig. 1A). Immunohistochemistry confirmed that newborn neurons in Pten-floxed mice infected with retroviruses expressing eGFP only (control) retained Pten immunoreactivity, whereas neurons in the same animal infected with a retrovirus expressing mCherry-T2A-Cre lacked Pten immunoreactivity (Pten KO, Fig. $1 B$, yellow asterisks). To analyze the emergence of hypertrophy after Pten KO, we compared the soma size in control versus Pten $\mathrm{KO}$ neurons within individual animals at several time points (DPI). Soma size was greater in Pten KO neurons by 7.5 DPI and at every time point thereafter (Fig. 1C: effects, age, $p<0.0001$; Pten KO, $p<0.0001$; by two-way repeated measures ANOVA with Bonferroni's post test, two-way repeated-measures ANOVA, neurons from 16 animals). In contrast, in a control experiment, Cre expression in non-Pten-floxed (i.e., "wild-type") C57BL/6J mice did not cause somatic hypertrophy $(115.0 \pm 2.7$ vs $115.6 \pm$ $1.4 \mu \mathrm{m}^{2}$ in control versus Cre-expressing neurons from 3 animals, $p=0.806$ by paired $t$ test).

Having validated that our system allowed the longitudinal examination of developing Pten KO neurons, we examined whether Pten deletion resulted in increased neuronal activity in vivo. In addition to inducing hypertrophy, Pten $\mathrm{KO}$ increases expression of the activity marker p-S6 (Pun et al., 2012). Indeed, in our system, we found that immunoreactivity for p-S6 was elevated in Pten KO neurons versus controls (24.5 DPI; Fig. 1D). Immunoreactivity of p-S6 was elevated in Pten KO neurons ( $p$ $<0.0001$ ), and this effect was significant at time points from 12.5 DPI onward (Fig. 1E; two-way repeated-measures ANOVA, neurons from 15 animals).

Although p-S6 is increased by activity, its levels are also increased in nonexcitable cells by Pten loss through disinhibition of the PI3K/AKT/mTOR/S6 kinase pathway; therefore, we examined levels of c-Fos, which also reports high levels of neuronal activity (Douglas et al., 1988; Knight et al., 2012). In Figure 1F, a Pten KO neuron (marked with ${ }^{\star *}$ ) is c-Fos-immunoreactive, whereas the control $\left(\right.$ marked $\left.^{\star}\right)$ is not (16.5 DPI). Compared with controls, overall c-Fos immunoreactivity in Pten KO neurons was significantly elevated $(p=0.0036)$, although for post hoc analyses of individual time points, this increase was significant only at 16.5 DPI (Fig. 1G; two-way repeated-measures ANOVA, neurons from 12 animals). Thus, expression levels of two activity markers provided evidence that developing Pten KO neurons were more active in vivo.

\section{Developing Pten KO neurons have altered electrophysiological properties}

Toward investigating the basis of increased activity in Pten KO cells, we tested how Pten altered the intrinsic properties of developing neurons. In whole-cell recordings, Pten $\mathrm{KO}$ was found to alter input resistance and membrane capacitance in response to a small voltage step $(40 \mathrm{~ms}, 10 \mathrm{mV}$; holding potential $-70 \mathrm{mV}$ ) (Fig. 2A). Capacitance increased with neuron age $(p<0.0001)$ but was higher in Pten KO cells $(p<0.0001)$, and this effect was significant at each DPI (Fig. 2B; two-way ANOVA, changes illustrated by Gaussian fit lines; 44 control, $37 \mathrm{KO}$ neurons). We found that, whereas input resistance normally decreased with age $(p<0.0001)$, Pten KO further reduced input resistance $(p$ $<0.0001$ ), and this effect was significant at all DPIs (Fig. 2C; two-way ANOVA, Gaussian fit lines; 43 control, 39 KO neurons).

We tested the developmental impact of Pten $\mathrm{KO}$ on firing threshold by measuring the current necessary to evoke action potentials ("rheobase"; 10 ms current step). Pten KO cells required much more current injection to reach spike threshold than did controls (Fig. $2 D$ ). In both control and Pten KO neurons, rheobase increased as cells matured $(p<0.0001)$ but was even greater in Pten KO cells ( $p$ $<0.0001$ ), and this effect was significant at DPI $\geq 12.5$ (Fig. $2 E$; two-way ANOVA; 42 control, 39 KO neurons; Gaussian fit). We used current-clamp recordings to measure action potential threshold $\left(\mathrm{V}_{\mathrm{m}}\right.$ at $10 \%$ of the peak of the first derivative of the spike waveform). Newborn granule neurons fired at increasingly more hyperpolarized membrane potentials as they matured $(p<0.0001)$, but action potential threshold in Pten KO neurons was at yet more hyperpolarized membrane voltages than in controls $(p$ $<0.0001$ ), and this effect was significant by 24.5 DPI (Fig. $2 F$; two-way ANOVA; 42 control, 39 KO neurons; Gaussian fit). Thus, although much more current was required to elicit action 
A Retroviral Constructs
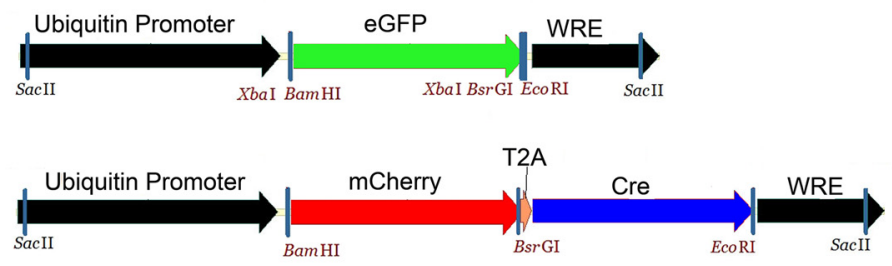

B Immunohistochemistry

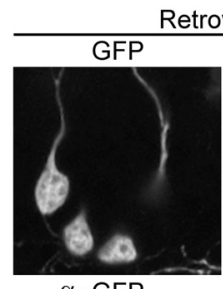

$\alpha-$ GFP

D

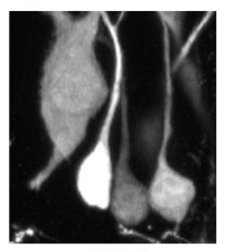

$\alpha-$ GFP

$\mathbf{F}$

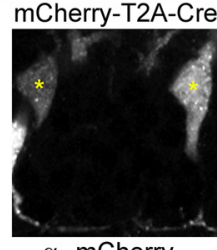

$\alpha-m$ Cherry

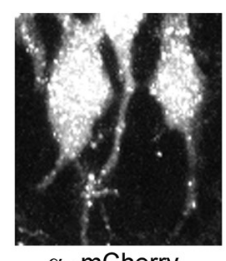

$\alpha-m$ Cherry

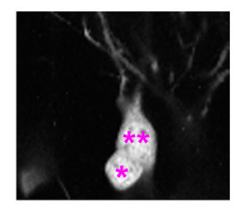

$\alpha-$ GFP

Control

Pten $\mathrm{KO}$

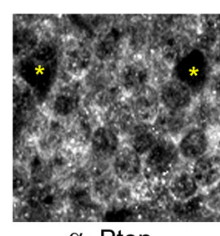

$\alpha$-Pten

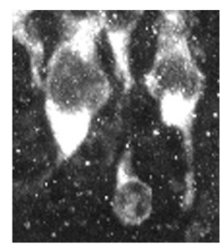

$\alpha-p S 6$

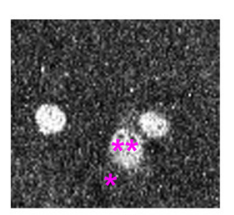

$\alpha-c$ Fos

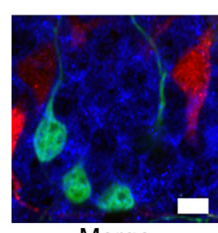

Merge

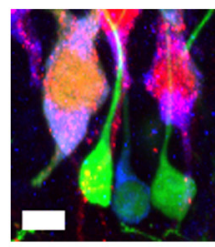

Merge

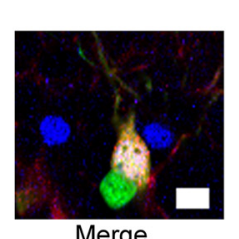

Neonatal Viral co-Injection

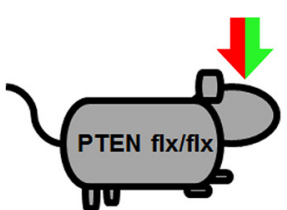

Dentate Gyrus

Acute Hippocampal Slice

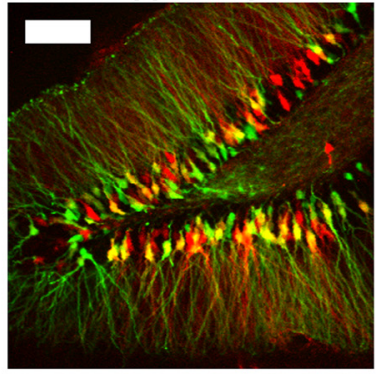

C
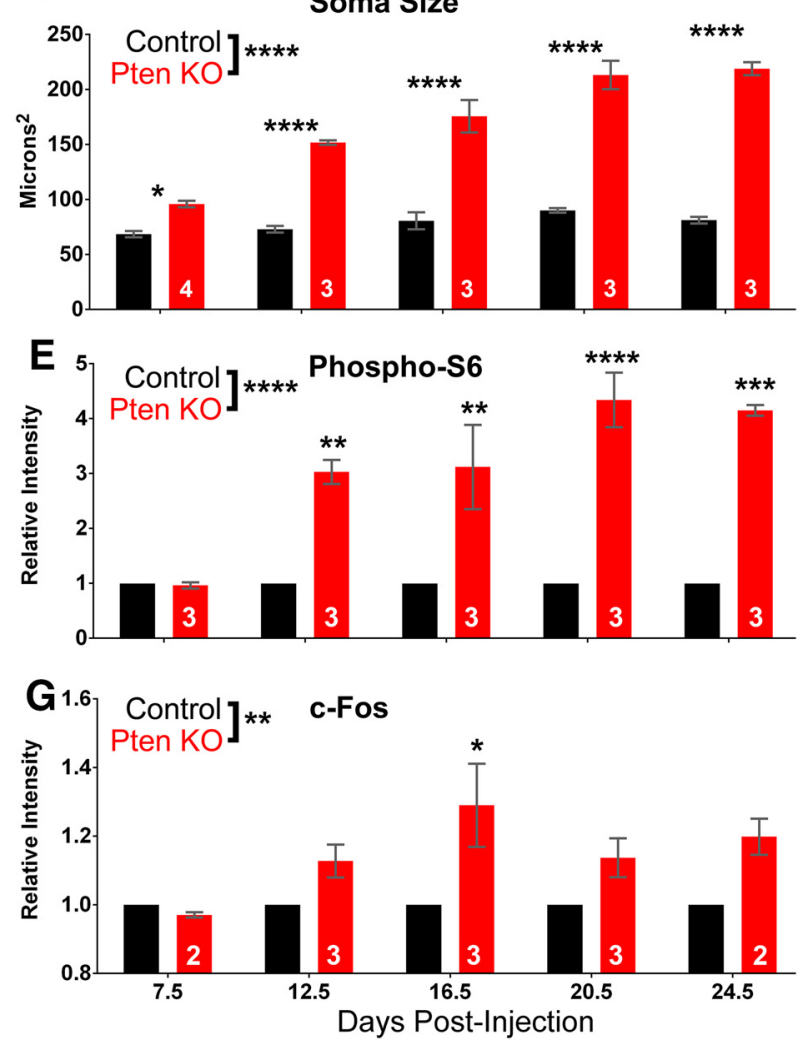

Figure 1. Retroviral deletion of Pten reveals early differences in neuronal morphology and activity. $\boldsymbol{A}$, Retroviruses encoding a fluorescent protein only, or a distinct fluorescent protein and Cre via a T2A element, are coinjected into Pten-floxed mice at P7. At increasing DPI, control versus Pten K0 dentate gyrus granule neurons are analyzed in acutely generated hippocampal slices (photomicrograph at 25 DPI). Scale bar, $100 \mu \mathrm{m}$. B, Immunolabeling Pten at 24.5 DPI shows that neurons expressing mcherry-T2A-Cre are Pten-depleted $\left({ }^{*}\right)$ versus control neurons expressing only eGFP. Scale bar, $20 \mu \mathrm{m}$. C, By analysis of tissue processed as in $\boldsymbol{B}$, Pten KO increased soma size ( $p<0.0001)$, and this effect was significant at each DPI. $\boldsymbol{D}$, Compared with control neurons, $\mathrm{p}$-S6 immunoreactivity is elevated in Pten K0 neurons in an example image at $24.5 \mathrm{DPI}$. Scale bar, $10 \mu \mathrm{m}$. $\boldsymbol{E}$, By analysis of tissue processed as in $\boldsymbol{D}$, Pten K0 elevates $p$-S6 immunoreactivity ( $p<0.0001$ ) compared with control neurons. This effect was significant after 7.5 DPI. F, Compared with a control neuron (*), a Pten K0 neuron (**) has increased immunoreactivity for c-Fos (16.5 DPI). Scale bar, $10 \mu \mathrm{m}$. G, Quantitation of c-Fos immunoreactivity reveals that Pten KO neurons have higher c-Fos immunoreactivity $(p=0.0036)$ compared with control neurons, and this effect was significant at 16.5 DPI. Data are mean \pm SEM; $n=$ animals. Asterisks next to brackets between control and "Pten K0" indicate overall significance of tw0-way repeated-measures ANOVA. Asterisks above bars indicate significance by Bonferroni's post-test. ${ }^{*} p<0.05 .{ }^{* *} p<0.01 .{ }^{* * *} p<0.001 .{ }^{* * *} p<0.0001$.

potentials in Pten KO neurons, they were initiated at lower membrane potentials.

In addition to these single-spike studies, we examined the current necessary to evoke multiple action potentials. In response to longer (500 ms) current steps, multiple-spike rheobase in Pten $\mathrm{KO}$ neurons was greater than for controls (Fig. 2G). We found that, although the current required to generate the peak spike frequency for each neuron rose with age $(p<0.0001)$, it was even higher in Pten KO neurons $(p<0.0001)$, and this effect was significant at DPI $\geq 12.5$ (Fig. $2 H$; two-way ANOVA; 32 control, $37 \mathrm{KO}$ neurons; quadratic fit). Thus, as for single spike threshold, Pten KO cells required much greater current injection to reach peak firing rate. However, we also examined Pten KO neurons, which were more active in terms of their spike rate, independent of the current injection necessary to evoke that spiking. Peak spike rate normally rose with age $(p<0.0001)$, but this indicator of excitability was yet higher in Pten KO neurons $(p<0.0228)$ (two-way ANOVA; 32 control, $37 \mathrm{KO}$ neurons; quadratic fit lines). Thus, although Pten $\mathrm{KO}$ neurons required greater current injection to fire, they fired at slightly more hyperpolarized membrane voltages and reached higher peak frequencies.

Having found that the firing rate of Pten $\mathrm{KO}$ cells was altered, we investigated whether the waveform of action potentials (as stimulated by the $500 \mathrm{~ms}$ current pulse) was also affected by Pten KO. Peak spike amplitude was found to rise with age (Tables 1 and $2 ; p=0.0015)$, but spike amplitude was consistently greater 
A

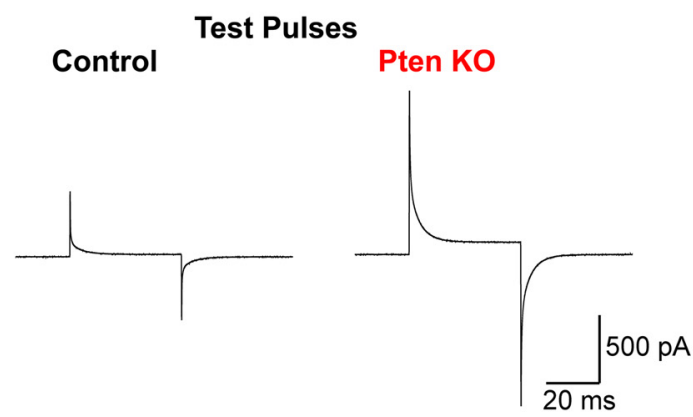

D
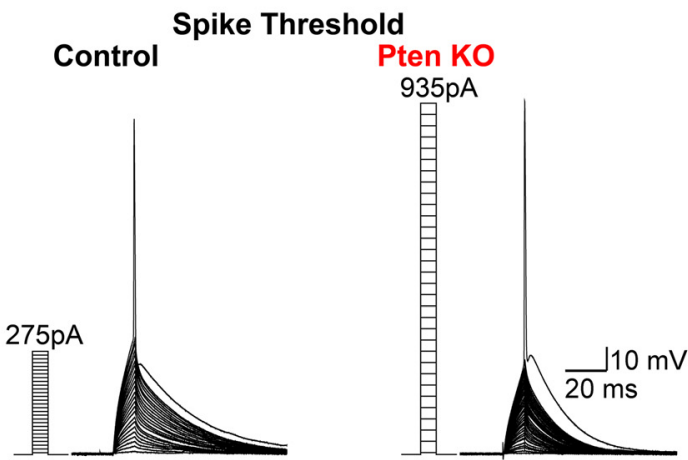

B
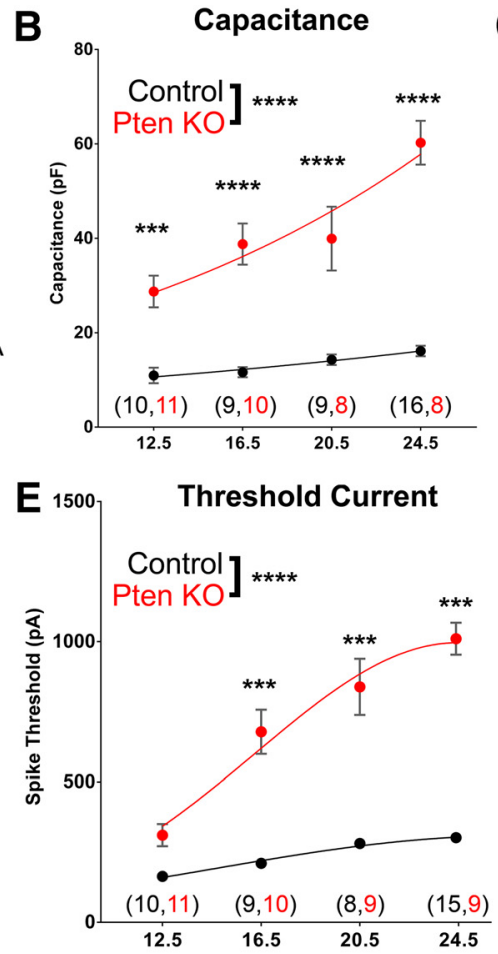

H
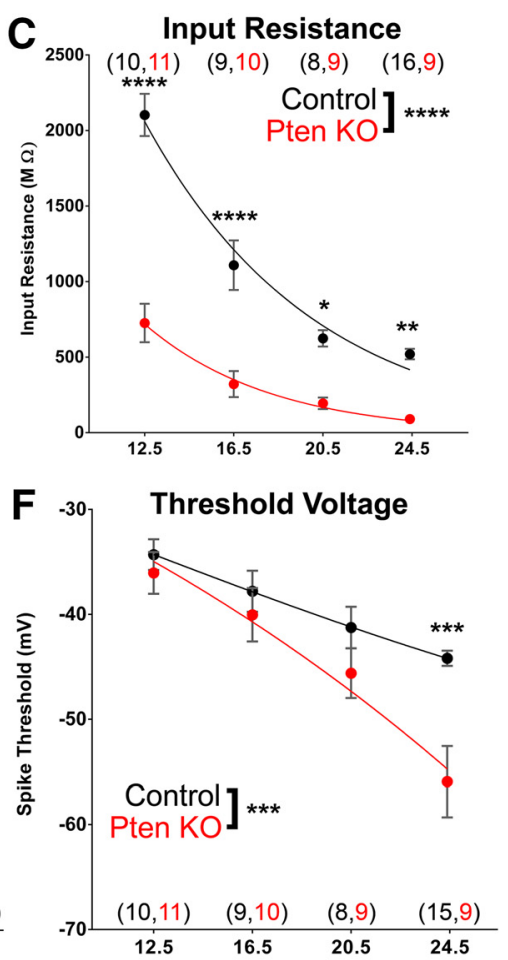

G

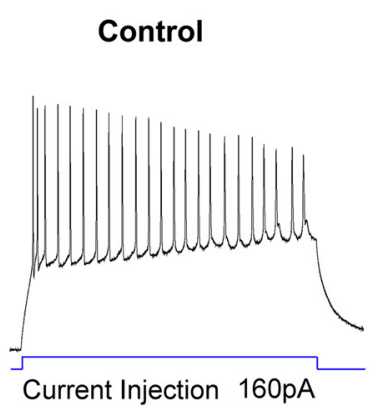

Spike Frequency

Pten KO

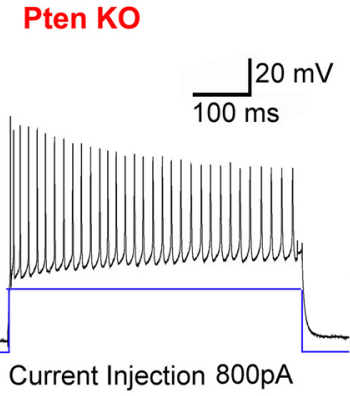

Peak Frequency Threshold

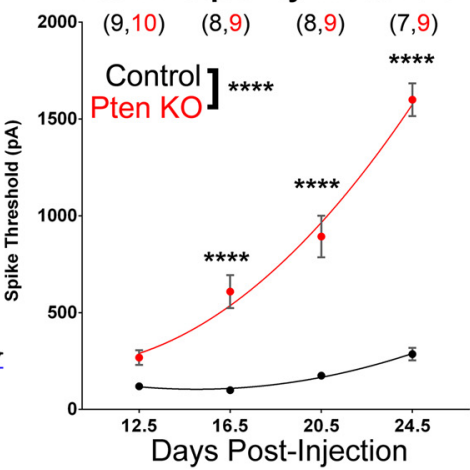

\section{I}

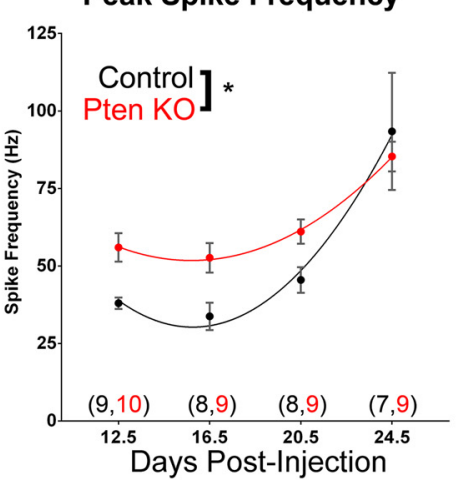

Figure 2. Retroviral deletion of Pten alters intrinsic properties of developing neurons. $A$, Pten K0 changed input resistance and cell capacitance; a Pten K0 neuron exhibited an exaggerated response to a test pulse (20.5 DPI). Capacitance increased $(\boldsymbol{B})$ and input resistance decreased $(\boldsymbol{C})$ with neuronal age $(p<0.0001, p<0.0001)$, but Pten K0 exaggerated these changes $(p<0.0001$, $p<0.0001$ ), and these effects of Pten KO were significant at each DPI. D, Spike threshold for a $10 \mathrm{~ms}$ current injection was greater in a Pten K0 than in a control neuron at 20.5 DPI. E, Current injection to elicit a spike rose as neurons matured $(p<0.0001)$, and Pten KO dramatically increased this trend $(p<0.0001)$; this effect was significant at DPI $\geq 12.5$ DPI. $F$, Neurons fired at more hyperpolarized potentials as they matured ( $p<0.0001$ ), but this was exaggerated by Pten KO ( $p=0.001$ ), with the effect of Pten K0 significant by 24.5 DPI. G, Spike trains from a 500 ms current injection show that a Pten $\mathrm{KO}$ neuron required more current to fire (20.5 DPI). $\mathbf{H}$, As neurons matured, peak firing frequency required increased current injection $(p<0.0001)$, and this was exacerbated by Pten KO $(p<0.0001)$, with the effect of Pten K0 reaching significance at DPI $\geq 12.5 . I$, Peak spike rate rose with age $(p<0.0001)$ but was higher with Pten K0 ( $p=0.0203)$. Data are mean $\pm \mathrm{SEM} ; n=$ neurons (indicated in bar bases). Asterisks next to brackets between control and "Pten K0" indicate overall significance of two-way ANOVA. Asterisks above bars indicate significance by Bonferroni's post-test. $\boldsymbol{B}-\boldsymbol{F}$, Gaussian fit lines. $\boldsymbol{H}$, I, Quadratic fit lines. ${ }^{*} p<0.05$. ${ }^{* *} p<0.005 .{ }^{* *} p<0.001 .{ }^{* * *} p<0.0001$.

in Pten $\mathrm{KO}$ neurons (Tables 1 and 2; $p=0.0009$ ); likewise, whereas action potentials had more rapid rise times (10\%-90\%) with increasing developmental age $(p=0.0004)$, rise time was more rapid in Pten KO cells $(p<0.0001)$. Whereas action potential halfwidth normally decreased with developmental age $(p<0.0001)$, it was longer in Pten $\mathrm{KO}$ cells $(p=0.0193)$. The integral of the afterhyperpolarization $\left(\mathrm{mV}^{\star} \mathrm{ms}\right)$ similarly decreased with developmental age of the neurons $(p<0.0001)$, but the afterhyperpolarization was diminished in Pten KO cells $(p=0.0193)$.

Pten KO increases sensitivity to afferent stimulation

Although intrinsic electrophysiological properties were altered by Pten $\mathrm{KO}$, the increased activity of Pten $\mathrm{KO}$ neurons in vivo could also be due to altered responsiveness to synaptic drive. We therefore tested whether Pten KO caused developing granule neurons to be more active in response to stimulation of the presynaptic perforant path. In example traces (20.5 DPI; Fig. $3 A$ ), although the Pten $\mathrm{KO}$ neuron required much more direct current injection to reach threshold than did a control (as in Fig. 2), the Pten KO neuron fired more, and at lower intensities of perforant path stimulation than did the neighboring control cell. This suggested that Pten KO cells required more current to charge their larger membrane surface area but were nonetheless more sensitive to depolarizing synaptic input. With cells from 20.5 to 24.5 DPI, we found (as expected) for all cells spike probability rose with increased levels of afferent stimulation intensity $(p<0.0001)$, 
Table 1. Action potential waveform is altered in Pten knockout neurons ${ }^{a}$

\begin{tabular}{|c|c|c|c|c|c|c|c|c|}
\hline & \multicolumn{2}{|l|}{$12.5 \mathrm{DPI}$} & \multicolumn{2}{|l|}{$16.5 \mathrm{DPI}$} & \multicolumn{2}{|l|}{$20.5 \mathrm{DPI}$} & \multicolumn{2}{|l|}{$24.5 \mathrm{DPI}$} \\
\hline & Mean & SEM & Mean & SEM & Mean & SEM & Mean & SEM \\
\hline \multicolumn{9}{|c|}{ Action potential amplitude, mV } \\
\hline Control & 73.4 & 1.5 & 71.9 & 3.8 & 78.6 & 3.0 & 84.9 & 3.6 \\
\hline Pten KO & 78.8 & 2.9 & 83.4 & 3.0 & 86.5 & 3.2 & 89.8 & 2.8 \\
\hline \multirow[t]{2}{*}{$p$} & 0.6427 & & 0.0515 & & 0.2608 & & $>0.9999$ & \\
\hline & \multicolumn{8}{|c|}{ Effects: age $(p=0.0015), \operatorname{Pten} \mathrm{KO}(p=0.0009)$, interaction $(p=0.6968)$} \\
\hline \multicolumn{9}{|c|}{ Action potential rise time, $\mathrm{ms}$} \\
\hline Control & 0.142 & 0.007 & 0.148 & 0.009 & 0.117 & 0.006 & 0.097 & 0.006 \\
\hline Pten KO & 0.100 & 0.007 & 0.102 & 0.011 & 0.093 & 0.004 & 0.090 & 0.005 \\
\hline \multirow[t]{2}{*}{$p$} & 0.0002 & & 0.0002 & & 0.1257 & & $>0.9999$ & \\
\hline & \multicolumn{8}{|c|}{ Effects: age ( $p=0.0004)$, Pten KO $(p<0.0001)$, interaction $(p=0.0464)$} \\
\hline \multicolumn{9}{|c|}{ Action potential half-width, ms } \\
\hline Control & 0.670 & 0.029 & 0.642 & 0.029 & 0.610 & 0.011 & 0.585 & 0.016 \\
\hline Pten KO & 0.773 & 0.025 & 0.724 & 0.036 & 0.662 & 0.046 & 0.566 & 0.025 \\
\hline \multirow[t]{2}{*}{$p$} & 0.0451 & & 0.2583 & & 0.9298 & & $>0.9999$ & \\
\hline & \multicolumn{8}{|c|}{ Effects: age $(p<0.0001)$, Pten KO ( $p=0.0193)$, interaction $(p=0.1731)$} \\
\hline \multicolumn{9}{|c|}{ Afterhyperpolarization integral, $\mathrm{mV}^{*} \mathrm{~ms}$} \\
\hline Control & -102.57 & 9.39 & -58.94 & 11.25 & -46.88 & 8.60 & -33.42 & 8.5 \\
\hline Pten KO & -74.26 & 13.01 & -35.82 & 14.25 & -36.37 & 13.53 & -19.54 & 8.36 \\
\hline \multirow[t]{2}{*}{$p$} & 0.2582 & & 0.6346 & & $>0.9999$ & & $>0.9999$ & \\
\hline & \multicolumn{8}{|c|}{ Effects: age $(p<0.0001)$, Pten KO ( $p=0.0193)$, interaction $(p=0.0193)$} \\
\hline
\end{tabular}

${ }^{a}$ Action potentials were elicited by a $500 \mathrm{~ms}$ current injection in control and in Pten $\mathrm{KO}$ neurons, and the kinetics and amplitude of the resultant waveform were analyzed. Action potential amplitude increased with the developmental age of neurons but was consistently higher in Pten $\mathrm{KO}$ neurons than in controls. Both action potential rise time and half-width decreased across the developmental window examined; however, rise time was decreased and half-width was increased by Pten KO. The afterhyperpolarization was also differentially regulated: although the afterhyperpolarization integral (mV*ms) diminished with developmental age, it was greater in control cells than in Pten KO cells.

Table 2. Sample sizes for action potential waveform analyses

\begin{tabular}{lcr}
\hline & Neurons & \\
\cline { 2 - 3 } DPI & Control & K0 \\
\hline 12.5 & 9 & 10 \\
16.5 & 8 & 9 \\
20.5 & 8 & 9 \\
24.5 & 7 & 9 \\
Total & 32 & 37 \\
\hline
\end{tabular}

but that Pten $\mathrm{KO}$ cells exhibited higher spike probabilities than did controls $(p=0.0004)$, and this effect was significant at afferent stimulation intensities $\geq 0.150 \mathrm{~mA}$ (Fig. $3 B$; two-way repeated-measures ANOVA; 15 control, 12 KO neurons; cumulative Gaussian line).

Because our electrophysiology suggested that one mechanism increasing activity in Pten KO neurons was an increased sensitivity to depolarizing synaptic input, we used calcium imaging to confirm this finding. We generated a novel retrovirus expressing GCaMP6s (Chen et al., 2013) and coinjected this with our mCherry-T2A-Cre retrovirus to make a subset of GCaMP6s cells also Pten KOs (Fig. 3C). Using multiphoton microscopy, we distinguished controls expressing GCaMP6s only $\left(^{*}\right)$ from Pten KO cells expressing GCaMP6s and mCherry-T2A-Cre ${ }^{* *}$ ) (Fig. 3D; 20.5 DPI). At 20.5-24.5 DPI, using epifluorescence, we measured somatic GCaMP6s in control and Pten KO cells at 30 frames per second while stimulating the perforant path $(3 \times$, every $13 \mathrm{~s}: 1 \mathrm{~ms}$ pulses at $20 \mathrm{~Hz}$ for $500 \mathrm{~ms}$ ). An image montage and associated plots of somatic intensity changes (Fig. $3 E$, top) demonstrate that Pten KO neurons had greater GCaMP6s transients for any given perforant path stimulation intensity than did controls (Fig. 3E, bottom). As with spike probability, background-corrected relative increases in GCaMP6s intensity (Peak $\Delta \mathrm{F}_{\text {Rea }} 1 / \mathrm{F}_{0}$ ) (Chen et al., 2013) increased with stimulation intensity for all cells ( $p$ $<0.0001)$, but Pten KO further increased evoked Peak $\Delta \mathrm{F}_{\text {Real }} / \mathrm{F}_{0}$ $(p=0.0263)$, and this effect was significant at stimulation intensities $\geq 0.200 \mathrm{~mA}$ (Fig. 3F; two-way repeated-measures ANOVA;
10 control, $11 \mathrm{KO}$ neurons; quadratic curves). Thus, both calcium imaging and whole-cell recordings showed that Pten KO neurons fired more readily than did controls in response to equivalent afferent stimulation.

\section{Developing Pten KO neurons have increased dendritic outgrowth and protrusions}

Why were Pten KO neurons more responsive to afferent input, even though they required more inward current to fire? One possibility is that increased synaptogenesis in Pten KO neurons leads to greater synaptic drive. Using live multiphoton microscopy of Pten KO and control neurons in acute hippocampal slices, we quantified dendritic protrusions in the outer molecular layer, the territory of lateral perforant path inputs, between 12.5 and 24.5 DPI. Because rapid photobleaching of mCherry made it impractical for imaging protrusions, we visualized protrusions using eGFP. Control neurons (30, from 11 animals) were those expressing only eGFP in wild-type or Pten-floxed mice. Pten KO neurons (42, from 13 animals) were those expressing eGFP-T2ACre in Pten-floxed mice (Fig. 4A). During development, the density of dendritic protrusions increased $(p=0.0011)$, but Pten KO further increased protrusion density $(p<0.0001)$, and this effect was significant at each DPI (Fig. $4 B$; two-way ANOVA; 30 control, $42 \mathrm{KO}$ neurons). We found that, in addition to being more numerous, the dendritic protrusions in these same cells had a greater mean length with Pten KO $(p<0.0001$, two-way ANOVA; Table 3 and 4). Furthermore, although dendrite caliber normally increased with age $(p=0.0115)$, this too was increased further by Pten KO ( $p<0.0001$, two-way ANOVA; Table 3 and 4).

Having found that there was a higher density of protrusions per dendrite in Pten KO neurons, we next measured how Pten KO altered overall dendrite morphology using Neurolucida reconstructions of tissue from 20.5 to 24.5 DPI. Pten KO caused somatic hypertrophy and dendritic outgrowth (representative reconstructions; Fig. 4C; 8 neurons were reconstructed for control, 8 for Pten KO). Pten KO increased the number of primary den- 
A

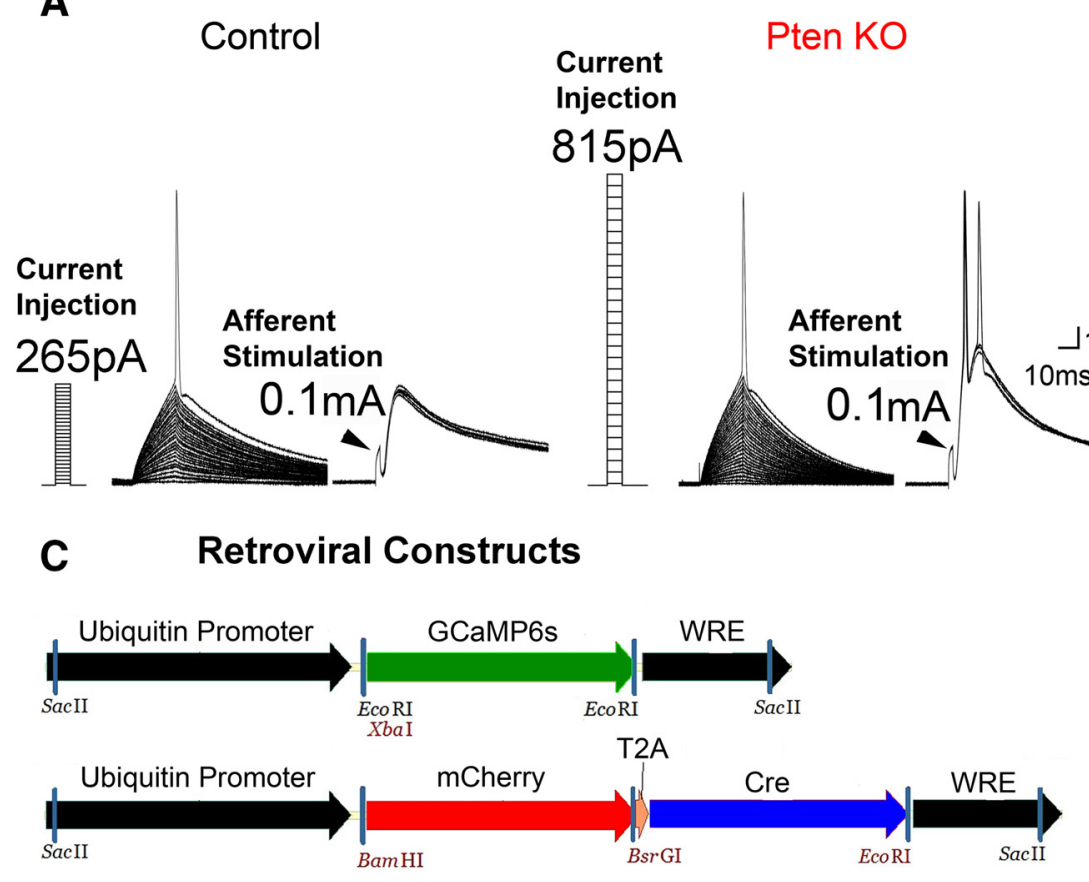

$\mathbf{E}$

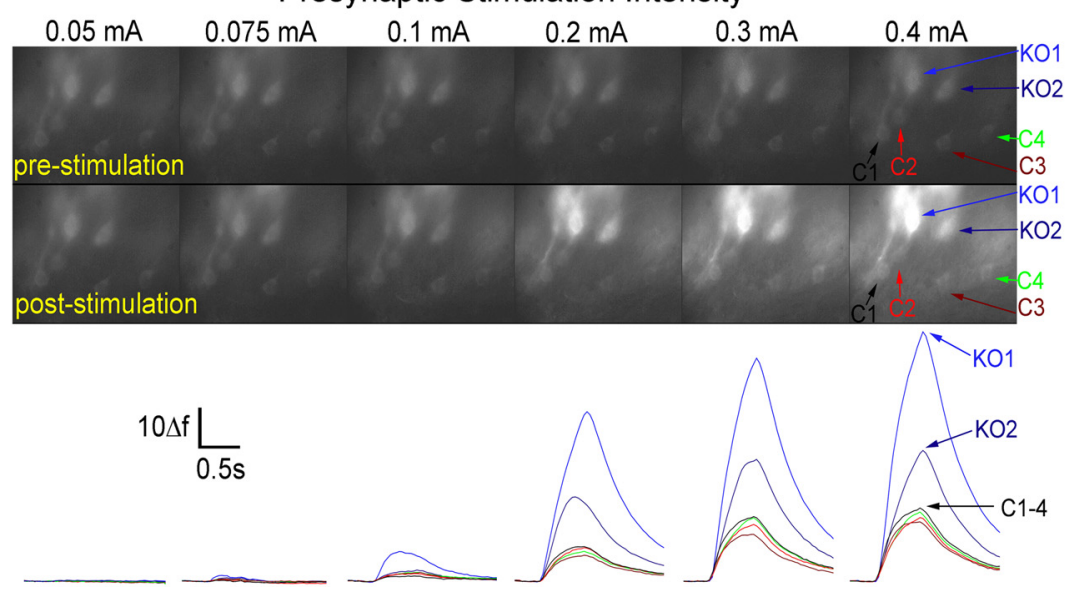

$10 \Delta f \underset{L .5 s}{\longleftarrow}$

Widefield Evoked GcAMP6s Transients

Presynaptic Stimulation Intensity
Single Spike Probability

B

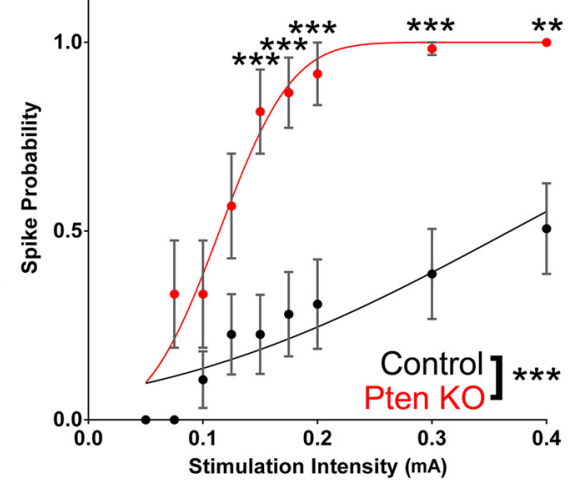

D Multi-Photon Imaging

Cre GCaMP6s Merge

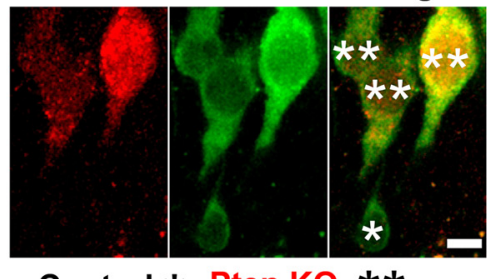

Control * Pten KO **

F Somatic GCaMP6s Signal

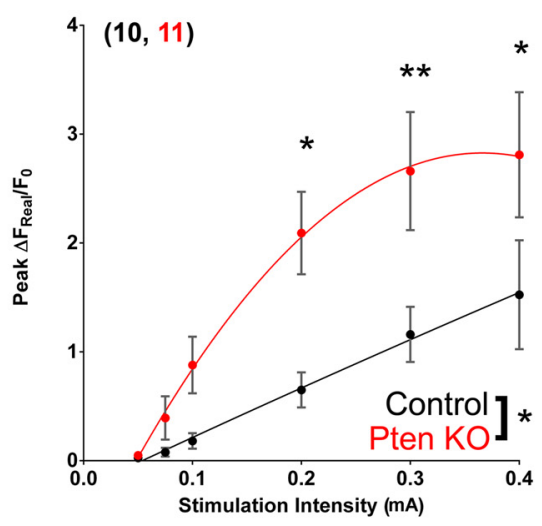

Figure 3. Pten KO increases developmental sensitivity to afferent stimulation. $A$, Although a Pten KO cell required greater current injection to fire than did a control ( $815 \mathrm{vs} 265 \mathrm{pA})$, the Pten KO cell fired at levels of presynaptic (perforant path) stimulation $(0.1 \mathrm{~mA})$, which did not elicit firing in the neighboring control cell (20.5 DPI). Arrowhead indicates the stimulus artifact. $\boldsymbol{B}$, Spike probability rose with perforant path stimulation intensity $(p<0.0001)$, but Pten K0 neurons were more likely to fire $(p=0.0004)$; this effect was significant at intensities $\geq 0.1 \mathrm{~mA}$. Cumulative Gaussian fit lines. C, Design of retroviruses to infect newborn granule neurons with the $\mathrm{Ca}^{2+}$ sensor GcAMP6s and/or mCherry-T2A-Cre. D, Multiphoton image of live 20.5 DPI neurons illustrating basal fluorescence of Pten $\mathrm{KO}$ neurons, which are those dual-infected with $\mathrm{mCherry}-\mathrm{T} 2 \mathrm{~A}-\mathrm{Cre}$ and GCaMP6s retroviruses (Pten K0, **), and of a control cell, which is infected with GCaMP6s retrovirus alone (control, ${ }^{*}$ ). Scale bar, $30 \mu \mathrm{m}$. E, Top, A montage demonstrates GcAMP6s fluorescence, detected by high-speed widefield imaging (30 FPS), in control (C1-4) or Pten K0 $(K 01,2)$ neurons before and after presynaptic perforant path stimulation at the indicated intensities. Bottom, Traces of $10 \times$ raw change in GcAMP6s intensity for the indicated cells. $\boldsymbol{F}$, The intensity of somatically recorded GcAMP6s fluorescence (Peak $\left.\Delta \mathrm{F}_{\text {Real }} / \mathrm{F}_{0}\right)$ rose with increasing afferent stimulation intensity $(p<0.0001)$, but Pten K0 neurons had greater evoked GcAMP6s transients $(p=0.026)$; this effect was significant at stimulation intensities $\geq 0.2 \mathrm{~mA}$. Data are mean $\pm \mathrm{SEM} ; n=$ neurons (indicated in bar bases). Asterisks next to brackets between control and Pten $\mathrm{KO}$ indicate overall significance of two-way repeated-measures ANOVA. Asterisks above bars indicate significance by Bonferroni's post-test. $\boldsymbol{B}, \boldsymbol{F}$, Quadratic fit lines. ${ }^{*} p<0.05 .{ }^{* *} p<0.005$. ${ }^{* *} p<0.001$.

drites originating from the soma (dendrites, $p=0.0008)$, the number of dendritic branching points (nodes, $p<0.0001$ ), the number of distal dendrite branches (ends, $p=0.0002$ ), the total dendritic length $(p<0.0001)$, the total dendrite surface area $(p=0.0001)$, and the total dendrite volume $(p=0.0016)$ (Fig. $4 D$; $t$ tests; 8 control, $8 \mathrm{KO}$ neurons). Thus, in addition to increas- ing the density of dendritic protrusions, Pten KO increased the total number and territory of primary dendrites.

The increases in total dendritic protrusions could contribute to Pten KO cell's increased sensitivity to synaptic input if the protrusions are functional synapses. We therefore performed immunohistochemistry on control neurons (expressing GFP only; 


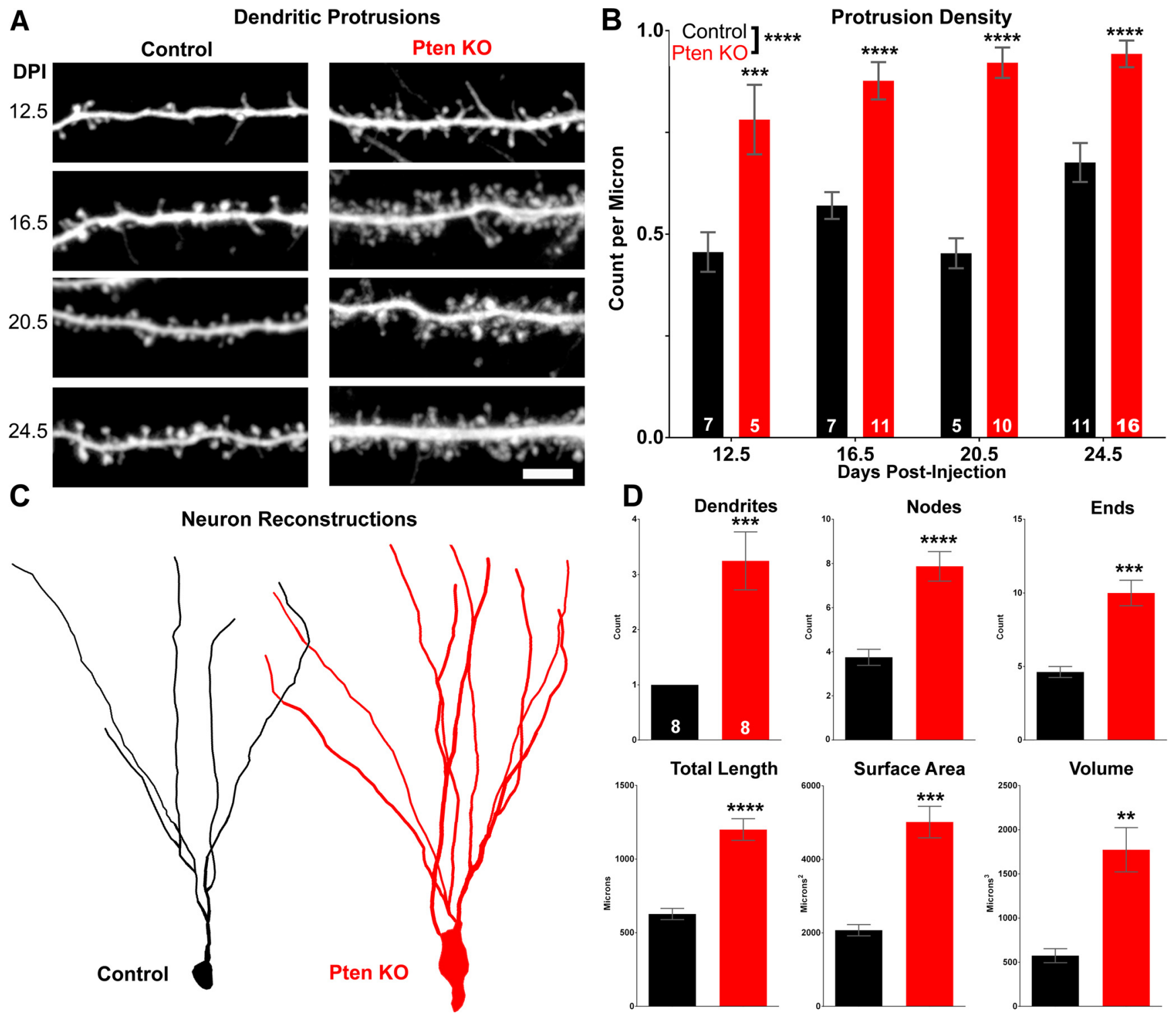

Figure 4. Developing Pten KO neurons have more dendritic protrusions and more dendritic arborization than do controls. $A$, Multiphoton microscope images of live distal dendrite segments of control or Pten K0 granule neurons demonstrate that, although dendritic protrusion density increases with neuron age, Pten K0 neurons consistently had more protrusions. Scale bar, $10 \mu \mathrm{m}$. $\boldsymbol{B}$, Quantifying the protrusion density changes documented in $\boldsymbol{A}$, protrusion density increased with age ( $p=0.001$ ), but Pten K0 further increased protrusion density ( $p<0.0001$ ); this effect was significant at each DPI. C, From fixed tissue, Neurolucida reconstructions of control and Pten KO neurons at 24.5 DPI demonstrate a grossly altered dendritic architecture. D, Quantifying the morphological differences as in $\boldsymbol{C}$. The number of dendrites, branching points (nodes), and dendrite ends were increased in Pten K0 neurons at 20.5-24.5 DPI $(p=0.0008, p<0.0001$, and $p=$ 0.0002 , respectively). Likewise, total dendrite length, surface area, and volume were all also increased in Pten KO neurons $(p<0.0001, p=0.0001$, and $p=0.0016$, respectively). Data are mean \pm $\mathrm{SEM} ; n=$ neurons (indicated in bar bases). $\boldsymbol{B}$, Asterisks next to brackets between control and Pten K0 indicate overall significance of two-way ANOVA. Asterisks above bars indicate significance by Bonferroni's post-test. $\boldsymbol{D},{ }^{* *} p<0.005$ ( $t$ test). ${ }^{* * *} p<0.001$ ( $t$ test). ${ }^{* * *} p<0.0001$ ( $t$ test).

pRubi) and on Pten KO neurons (expressing GFP and Cre; pRubi-GFP-T2A-Cre), both in Pten-floxed mice, at 24.5 DPI. Immunoreactivity for GFP defined infected neurons $(\alpha$-GFP; Fig. 5A, $C$, left panels). Immunoreactivity for the glutamatergic postsynaptic density scaffolding protein, $\mathrm{SH} 3$ and multiple ankyrin repeat domains protein 2 ( $\alpha$-Shank2; Fig. $5 A, C$, second panels) was used to identify postsynaptic sites. Presynaptic sites were labeled using an antibody against the vesicle associated membrane protein, synaptobrevin 2 ( $\alpha$-SynB; Fig. $5 A, C$, third panels). Images were thresholded to the $\alpha$-GFP signal, delimiting the dendrite and dendritic protrusions of the infected neurons. Using this region of interest, immunoreactivity for SynB and Shank 2 could be colocalized to the infected neurons (Fig. $5 B, D$ ). Under these conditions, we could clearly detect both the presyn- aptic and postsynaptic markers either adjacent to, or colocalized at, the dendritic protrusions of both control and Pten KO neurons (mushroom-type spines; Fig. 5B,D, insets). These results suggested that the dendritic protrusions of both control and Pten KO neurons are functional synapses; we therefore investigated whether Pten KO neurons developed increased synaptic input.

Pten KO neurons have increased excitatory synaptic currents More dendrites, greater surface area, and increased density of dendritic protrusions could be a physical substrate and, thus, the mechanism for, the increased sensitivity to afferent path stimulation of Pten $\mathrm{KO}$ neurons. We therefore compared evoked synaptic currents ( 5 stimulations, 1 every $13 \mathrm{~s}: 0.1 \mathrm{~ms}, 0.2 \mathrm{~mA}$ ) in 
Table 3. Pten knockout produces developmental changes in dendritic protrusion architecture ${ }^{a}$

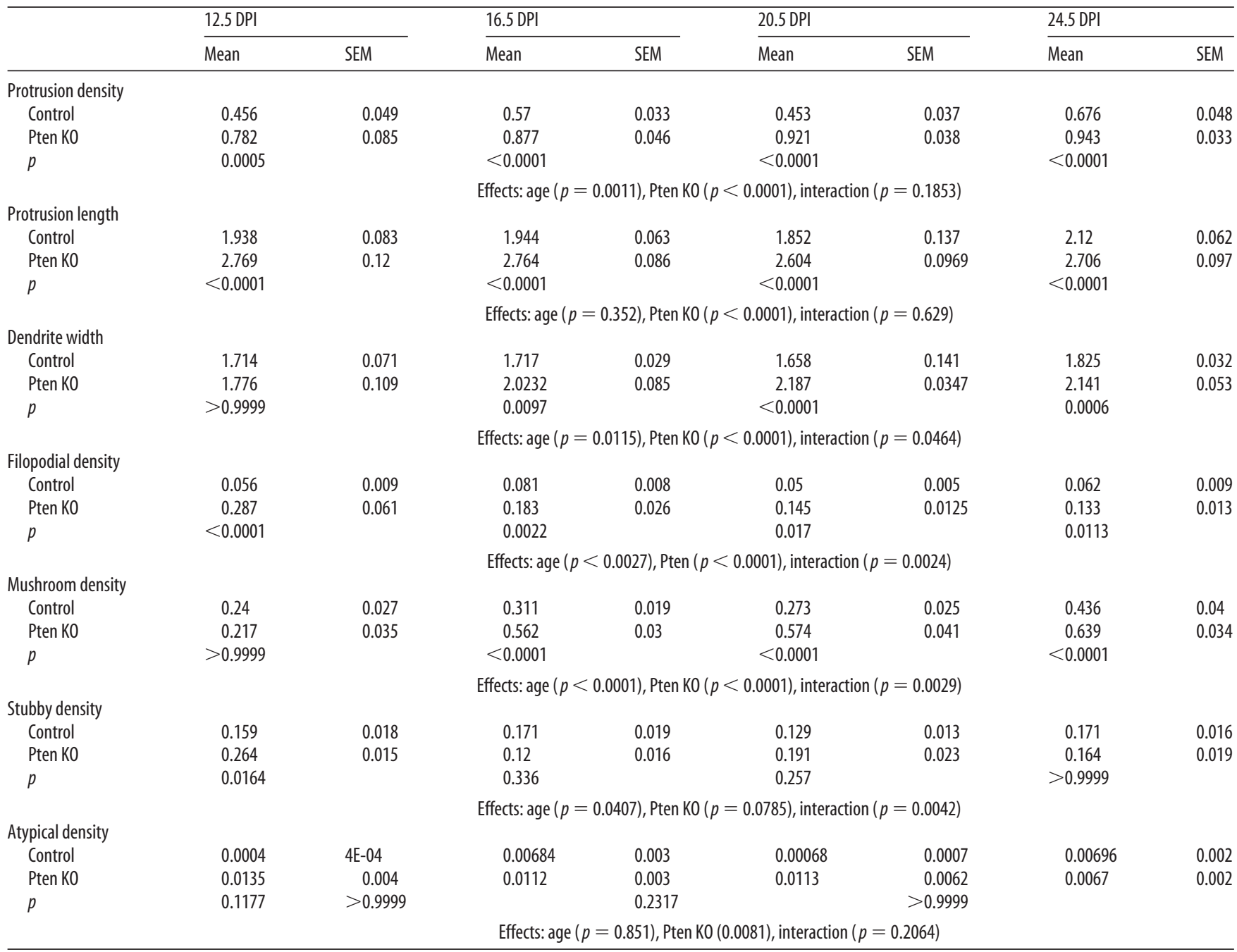

${ }^{a}$ Analysis of multiphoton time-lapse z-stacks of retrovirus-infected dentate gyrus granule neuron dendrites within the outer molecular layer reveals that Pten knockout causes developmental differences in dendritic protrusions. Overal density of protrusions was increased, as was the length of these protrusions and the width of the dendrites from which they originated. Persistently, there was an increased abundance of thin/filopodial protrusions in Pten KO cells; later in development, Pten KO neurons also had a higher density of mushroom spines than did controls. Although stubby spine density did not alter Pten K0, atypical spine density was increased in the cells compared with controls.

Table 4. Sample sizes for dendritic protrusion architecture analyses

\begin{tabular}{llllll}
\hline & & & & & Pten K0 \\
\cline { 6 - 6 } \cline { 5 - 6 } DPI & Neurons & Animals & & Neurons & Animals \\
\hline 12.5 & 7 & 2 & 5 & 2 \\
16.5 & 7 & 3 & 11 & 4 \\
20.5 & 5 & 2 & 10 & 4 \\
24.5 & 11 & 4 & 16 & 3 \\
Total & 30 & 11 & 42 & 13 \\
\hline
\end{tabular}

control versus Pten KO neurons. Mixed AMPA and GABA A $_{\text {p }}$ postsynaptic currents were recorded at $-90 \mathrm{mV}$. The contribution of AMPA-mediated excitatory currents was determined by subtracting the SR95531 (Gabazine, $10 \mu \mathrm{M}$ )-sensitive GABA $_{\mathrm{A}}$, IPSC component (Fig. 6A). We found that Pten $\mathrm{KO}$ did not affect $\mathrm{GABA}_{\mathrm{A}}$ current amplitude (Fig. $6 B ; p=0.419$; two-way ANOVA; 24 control, $33 \mathrm{KO}$ neurons). In contrast, Pten $\mathrm{KO}$ increased EPSC amplitude (Fig. 6C; $p=0.0003$; two-way ANOVA; 24 control, 33 $\mathrm{KO}$ neurons). This suggested that Pten $\mathrm{KO}$ disproportionately increased synaptic excitation. Quantifying this, we found that, whereas the EPSC/IPSC amplitude ratio increased as neurons matured ( $p=0.0043$ ), Pten KO further increased the EPSC to IPSC amplitude ratio $(p=0.0018)$, and this effect was significant by
24.5 DPI (Fig. 6D; two-way ANOVA; 24 control, $33 \mathrm{KO}$ neurons). Therefore, in developing Pten $\mathrm{KO}$ neurons, the excitatory postsynaptic responses were greater than they were in control cells.

We next determined whether the increased EPSC amplitude in Pten KO cells was due to a greater number of synaptic contacts or was due to stronger individual synapses. Replacement of extracellular calcium with strontium desynchronizes presynaptic vesicle release, generating aEPSCs that resemble quantal events (Bekkers and Clements, 1999; Rudolph et al., 2011). We did not find a significant difference in the degree to which the amplitude of the synchronous component of the evoked release was decreased after the addition of strontium-containing external solution. As in our previous experiments, in extracellular calcium, 20.4-24.5 DPI Pten KO neurons had larger evoked EPSCs (eEP$\mathrm{SCs}$ ) than did controls (Fig. $6 E, \mathrm{Ca}^{2+}$ traces). These events were smaller and desynchronized in a calcium-free, strontiumcontaining solution (Fig. $6 E, \mathrm{Sr}^{2+}$ traces), and we were able to detect aEPSCs (Fig. 6F, arrowheads). Mean aEPSC amplitude was larger in Pten KO neurons (Fig. 6G, averaged traces; 6I, histogram), but their kinetic properties were similar to controls (peakscaled, Fig. $6 H$ ), suggesting that the amount but not the type of depolarizing input was altered by Pten KO. As before, the aggregate eEPSC amplitudes in Pten KO neurons was much greater 


\section{Synapse Immunohistochemistry}

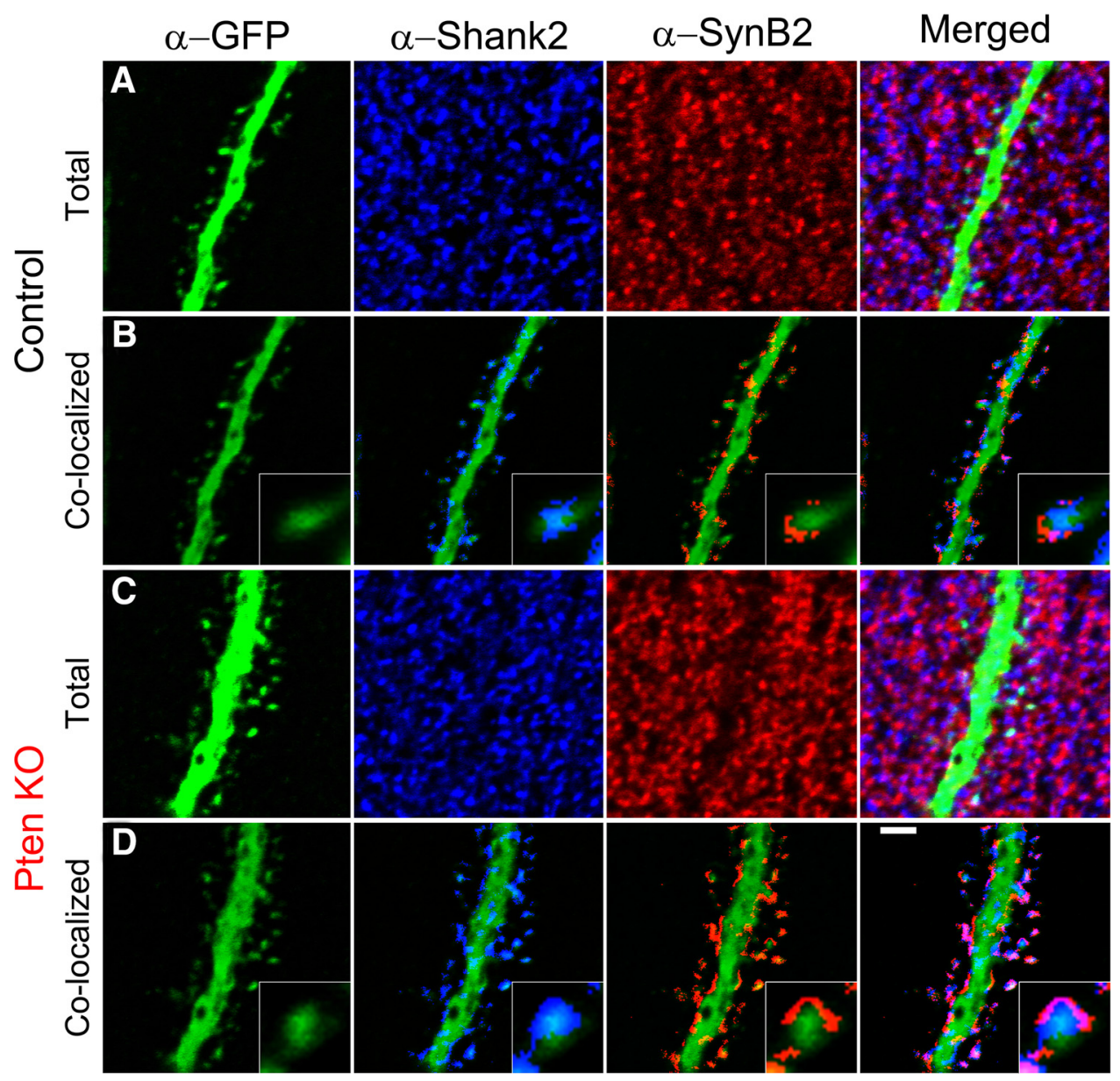

Figure 5. Dendritic protrusions have immunohistochemical markers of functional excitatory synapses. A, C, Dendrite segments of $24 \mathrm{DPI}$ control or Pten $\mathrm{KO}$ neurons (retroviral expression of GFP only or expression of GFP and (re, in Pten-floxed animals) visualized by confocal microscopy after immunohistochemical labeling of GFP (first panel). SH3 and multiple ankyrin repeat domain protein 2 (Shank2, second panel) was used to label the postsynaptic density of glutamatergic synapses. Presynaptic sites are labeled by the vesicle-associated membrane protein, Synaptobrevin 2 (SynB, third panel). B, D, GFP intensity is used as a threshold to define the territory of the infected neurons (first panel); ;in the territory of the infected neuron, Shank2 reactivity is found upon the dendritic shaft and dendritic protrusions (middle), whereas Syn2B is found in close apposition to or colocalized with Shank2 in these same domains (right), as evidenced in the mushroom spine inset images. Scale bar, $2 \mu \mathrm{m}$.

than in controls $(\sim 4.532$-fold, $p=0.0259$; Fig. $6 \mathrm{~J}$; $t$ test; 6 control, $6 \mathrm{KO}$ neurons). In contrast, quantal-like aEPSC amplitude in these same neurons was only 1.343 -fold greater in Pten $\mathrm{KO}$ neurons ( $p=0.0209$, Fig. $6 K$; $t$ test; 6 control, $6 \mathrm{KO}$ neurons). This suggested that the increased EPSC in Pten KO neurons was mostly due to an increased number of synaptic contacts onto Pten KO neurons and was modestly due to stronger individual synapses.

To quantify the increased synaptic drive, we estimated the number of quantal events (synaptic inputs) contributing to the evoked (0.2 $\mathrm{mA}$ stimulation) EPSC by dividing the amplitude of the eEPSC (in $\mathrm{Ca}^{2+}$ ) by the amplitude of the average aEPSCs (in $\mathrm{Sr}^{2+}$ ) for each neuron. We determined that there were, on average, 12.3 synaptic events per eESPC in controls, whereas there were 44.7 synaptic events per eEPSC in Pten KO neurons (3.63fold increase, $p=0.0322$; Fig. $6 L$; $t$ test; 6 control, $6 \mathrm{KO}$ neurons). If an increased release probability was principally responsible for the increased eEPSC, it might be expected that paired pulse ratio would be increased by a degree similar to the 3.63-fold increase in eEPSC. In post hoc analysis, we calculated that we had $99 \%$ power to detect as small as a 0.79 -fold change by two-tailed $t$ test using a significance level of 0.01 . Therefore, the increased eEPSC ampli- tude was likely a cell-autonomous effect because: it paralleled changes in dendritic protrusion density, the presynaptic cell was not genetically manipulated, and the paired pulse ratio was not different between control and Pten KO neurons ( $p=0.9193$; Fig. $6 M ; t$ test; 6 control, $6 \mathrm{KO}$ neurons).

The changes we observed with evoked currents strongly suggested an increase in the number and strength of excitatory synaptic inputs to Pten $\mathrm{KO}$ cells. To confirm this, we examined mEPSCs. Using control and Pten KO neurons from the 20.5-24.5 DPI range, cells were held at a potential of $-90 \mathrm{mV}$ in the presence of tetrodotoxin and SR-95531 (Gabazine). Spontaneous events were recorded using a potassium gluconate-based internal solution. Under these conditions, we could record quantal glutamatergic inputs as mEPSCs. The frequency and amplitude of individual mEPSCs were increased in Pten $\mathrm{KO}$ versus control cells (Fig. 7A, raw trace). The increased mEPSC amplitude was also evident in the average waveform of all mEPSCs recorded from Pten KO versus control neurons (Fig. $7 B$ ), although the kinetics were similar once peak scaled (Fig. 7C). Consistent with the increased number of dendritic protrusions and the greater number of aEPSCs per eEPSC, the frequency of mEPSC events was higher in Pten KO versus control cells $(p=0.0265$; Fig. $7 D$; 
A

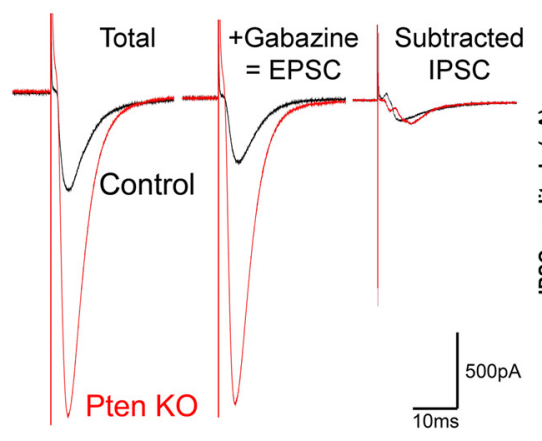

E
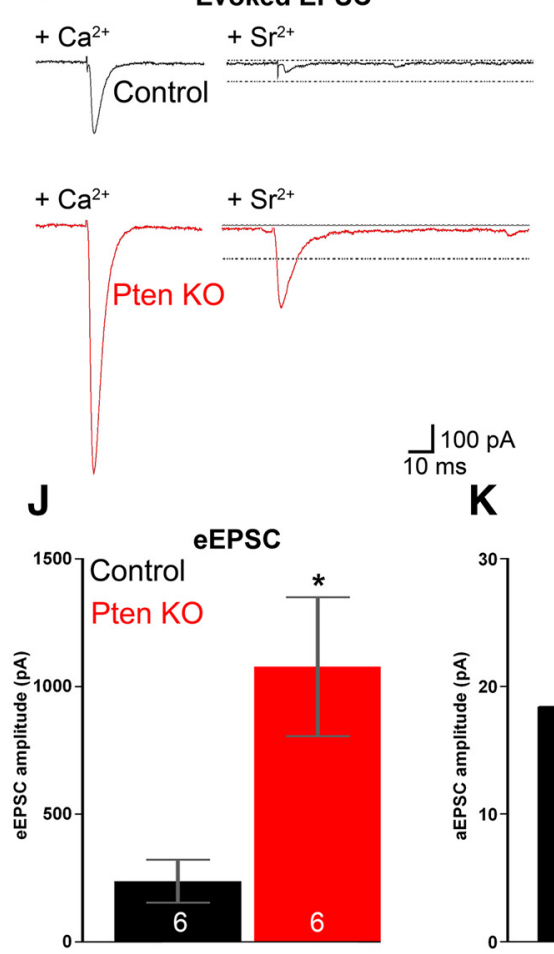

B Inhibitory Input C

F
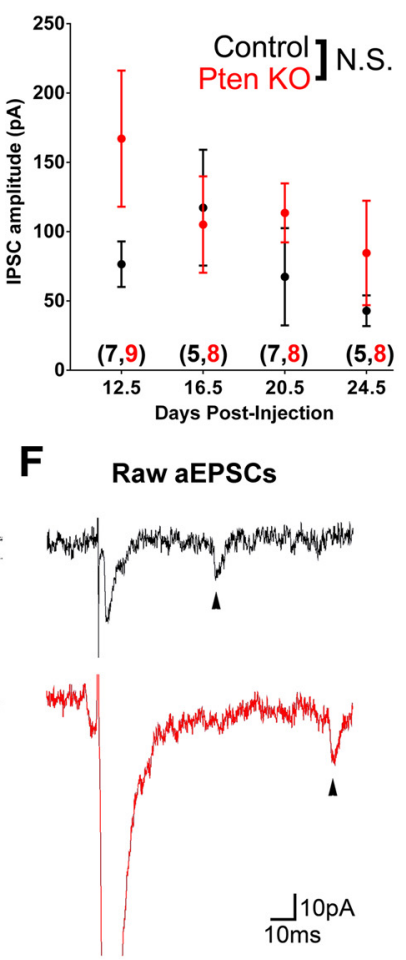

aEPSC

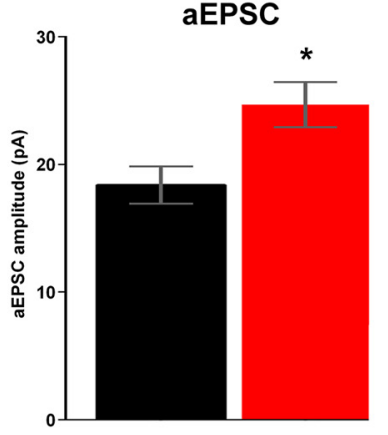

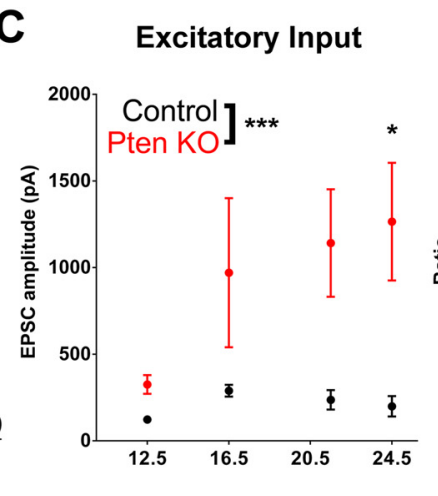

Excitatory Input

D Excitation:Inhibition

G Average aEPSC

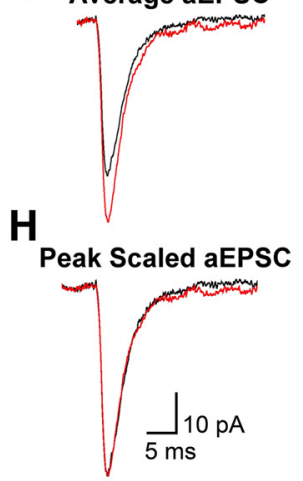

L aEPSC per eEPSC

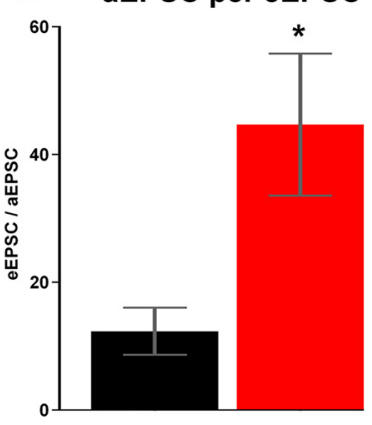

I

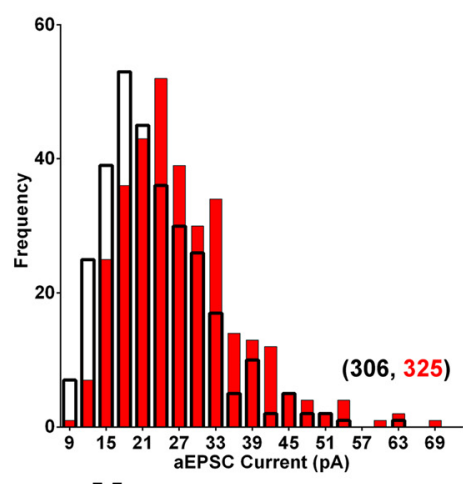

M

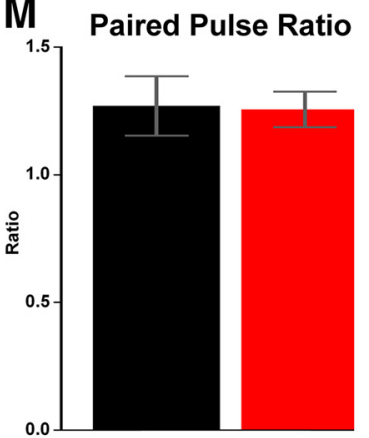

Figure 6. Developing Pten KO neurons have more excitatory synapses. $A$, Evoked synaptic currents are greater in a Pten KO versus control neuron (20.5 DPI). Blocking GABA $($ (SR95531) revealed a greater excitatory current (EPSC) in Pten K0, whereas the subtracted inhibitory current (IPSC) was similar. $\boldsymbol{B}$, Neither age $(p=0.419)$ nor Pten K0 ( $p=0.115)$ changed IPSC amplitude. C, Pten KO ( $p=0.0003)$ nor age $(p=0.167)$ increased EPSC amplitude. D, EPSC to IPSC amplitude ratio increased with age $(p=0.0043)$, but Pten KO further increased EPSC to IPSC amplitude ratio $(p=0.0018) . E$, Compared with $\mathrm{Ca}^{2+}$-containing solution, evoked EPSCS (eEPSCs) in $\mathrm{Sr}^{2+}$ were asynchronous and smaller. $\boldsymbol{F}$, Quantal-like asynchronous EPSCs were detected in $\mathrm{Sr}^{2+}$ (aEPSCS, arrowheads; 10x scale from $\boldsymbol{E}$ ). G, Averaged aEPSCS were larger in Pten K0 cells. $\boldsymbol{H}$, Peak-scaled averaged aEPSCs from control or Pten K0 neurons had similar kinetics. I, Histogram: amplitude of aEPSC currents, which were greater in Pten KO versus control neurons. J, Mean eEPSC amplitude, recorded in Ca ${ }^{2+}$, was greater in Pten KO versus control neurons $(p=0.0259)$. $\boldsymbol{K}$, Mean aEPSC amplitude, recorded in $\mathrm{Sr}^{2+}$, was slightly higher in Pten KO neurons $(p=0.0209)$. $\boldsymbol{L}$, Pten KO neurons had more aEPSCS per eEPSC $(p=0.0322) . M$, The paired pulse ratio was similar between control and Pten KO neurons $(p=0.9193)$. Data are mean \pm SEM; $n=$ neurons (indicated in bar bases), except in $I$, where $n=\mathrm{aEPSC}$, indicated on graphs. $\boldsymbol{B}-\boldsymbol{D}$, Asterisks next to brackets between control and "Pten K0" indicate overall significance of two-way ANOVA. Asterisks above bars indicate significance by Bonferroni's post-test. $I-L$, Asterisks indicate significance by $t$ test. ${ }^{*} p<0.05$. ${ }^{* *} p<0.005$. ${ }^{* *} p<0.001$.

one-tailed $t$ test; $n=9$ control, $10 \mathrm{KO}$ neurons). Absent a change in the paired pulse ratio (i.e., Fig. $6 M$ ), the increased mEPSC frequency provides further support to the interpretation that there are a greater number of synapses upon Pten KO cells (rather than an increased release probability at the same number of synapses). As with aEPSCs, the peak amplitude of mEPSCs was greater in Pten $\mathrm{KO}$ neurons than in controls $(p=0.0271$; Fig. $7 E$; one-tailed $t$ test; $n=9$ control, $10 \mathrm{KO}$ neurons). Although the kinetics of the peak-scaled mEPSC were visually similar between control and Pten KO neurons (i.e., Fig. 7B), quantification revealed a small but significantly increased $\mathrm{mESPC}$ rise time in Pten KO neurons versus controls ( $p=0.0045$; Fig. $7 F$; one-tailed $t$ test; $n=9$ control, $10 \mathrm{KO}$ neurons). In contrast, the mEPSC decay time was not significantly increased in Pten KO cells ( $p=0.1469$; Fig. 7G; one-tailed $t$ test; $n=9$ control, $10 \mathrm{KO}$ neurons).

Filopodia precede increased mushroom spine density in Pten KO neurons

Our data suggested that Pten $\mathrm{KO}$ cells were more active because they were more sensitive to afferent stimulation and that this increased sensitivity was due to an increase in the number of excitatory synapses Pten KO cells form. We therefore characterized synaptogenesis using live imaging in acute hippocampal slices. Dendritic protrusions in image series from the same neu- 
A

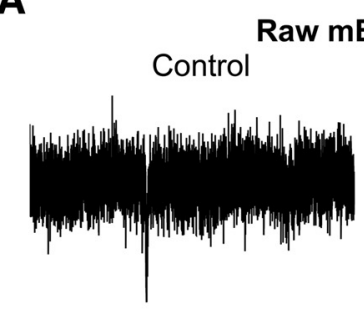

Raw mEPSCs

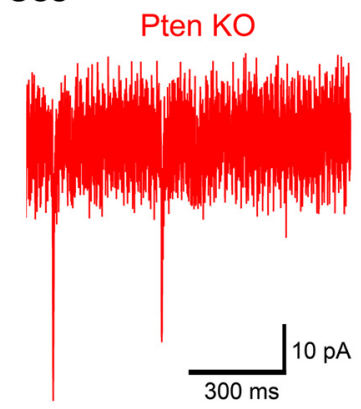

E

D

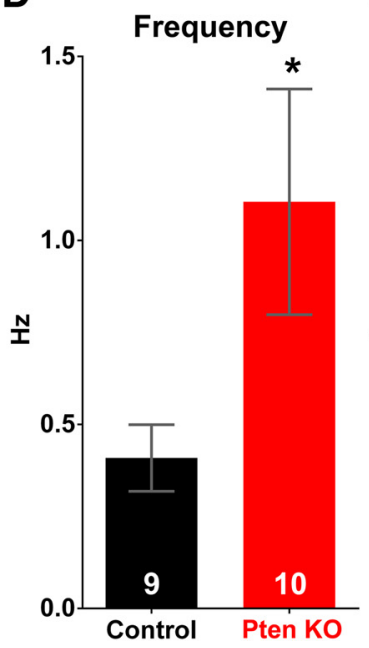

B
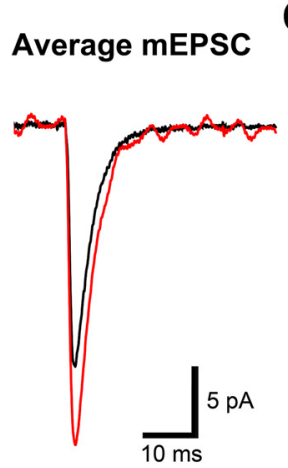

F

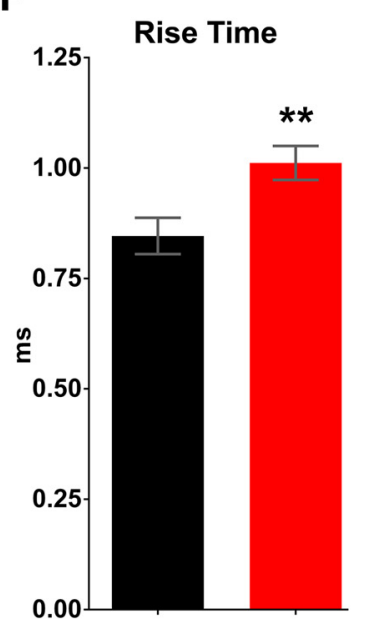

C

Peak Scaled mEPSC

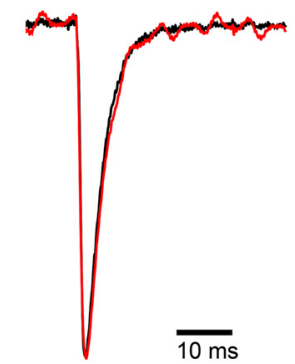

G

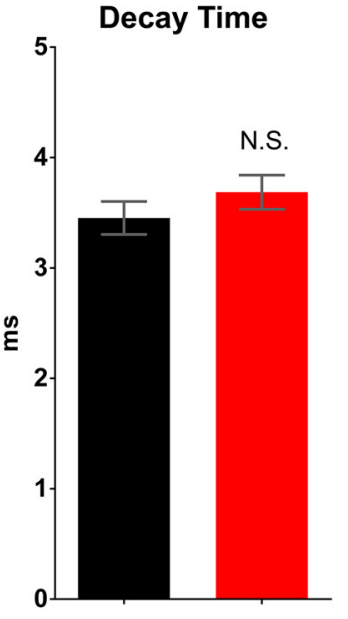

Figure 7. Excitatory quantal events are greater in size and frequency in Pten KO neurons. $A$, Example raw traces of mEPSCs recorded at 20.5 DPI from control and Pten KO neurons, illustrating the higher rate and peak amplitude of $\mathrm{mEPSC}$ events in Pten $\mathrm{KO}$ cells. $\boldsymbol{B}$, The average waveform of $\mathrm{mEPSC}$ (s recorded in Pten $\mathrm{KO}$ neurons has increased amplitude compared with $\mathrm{mEPSC}$ f from control neurons. $\boldsymbol{C}$, The peak-scaled mEPSC demonstrates similar kinetics between control and Pten $\mathrm{KO}$ conditions. $\boldsymbol{D}$, The frequency of $\mathrm{mEPSCs}$ is increased in Pten $\mathrm{KO}$ cells $(p=0.0265)$. $\boldsymbol{E}$, $\mathrm{mEPSCs}$ from Pten KO cells have a greater peak amplitude $(p=0.0271) . \boldsymbol{F}$, The mEPSC rise time is elevated by Pten KO $(p=0.0045)$. G, the mEPSC decay time is not significantly increased by Pten KO $(p=0$. 147). Asterisks indicate significance by $t$ test. ${ }^{*} p<0.05 .{ }^{* *} p<0.005$. N.S., Not significant.

rons in Figure $4 A, B$ were characterized as follows: thin/filopodial, stubby, mushroom, or atypical if not fitting another morphological category (example of classifications: Fig. 8A) (Peters and Kaiserman-Abramof, 1970; Arellano et al., 2007). The density of protrusions for each class was determined across development in control and Pten KO neurons.

We found that, whereas the density of thin/filopodial dendritic protrusions normally decreased with age $(p=0.0027)$, thin/filopodial density was markedly increased in Pten $\mathrm{KO}$ neurons $(p<0.0001)$, and this effect was significant at every DPI (Fig. 8B; Table 3 and 4; two-way ANOVA; 30 control neurons from 11 animals, 42 Pten KO neurons from 13 animals). Conversely, in these same neurons, although mushroom spine density normally increased with developmental age $(p<0.0001)$, it was even higher in Pten KO cells $(p<0.0001)$, and this effect was significant after $>12.5$ DPI (Fig. $8 C$; Table 3 and 4 two-way ANOVA). Therefore, a loss of Pten produces developmental increases in thin/filopodial dendritic protrusions that precede the increased density of mushroom spines seen later in Pten KO neurons. We also looked for potential changes in stubby and atypical spine densities: stubby spine densities did not differ with Pten KO $(p=0.0785$, Table 3 and 4 ), although atypical spine densities were higher in Pten KO neurons ( $p=0.0081$, Table 3 and 4$)$. Together, these morphological data suggest that Pten KO neurons form more mushroom (putatively functional) spines than controls, supporting the electrophysiological findings that indicated an increased number of glutamatergic synaptic sites.

Toward addressing why Pten $\mathrm{KO}$ neurons developed more spines, we quantified spine maturation as the frequency of instances in which a filopodial/thin protrusion became a mushroom spine. An example spine maturation is illustrated in Figure $8 D$ wherein a filopodial/thin protrusion (distinguished by a yellow asterisk) adopts a mushroom shape. Because the 20 min session only rarely captured maturation events, we pooled data across DPIs. Spine maturations per a given length of dendrite occurred more frequently in Pten $\mathrm{KO}$ neurons (Fig. $8 E$; $p=$ 0.0182 , $t$ test; 30 control, $42 \mathrm{KO}$ neurons). However, because total filopodial protrusion density was also higher in Pten KO neurons (i.e., Fig. 8B), we tested whether the proportion of protrusions that matured differed with Pten KO. We found that the proportion of protrusions that matured was not different between control and Pten KO neurons (Fig. 8F; $p=0.731$, $t$ test; 30 control, 42 KO neurons). Post hoc analysis revealed that this experiment had $99 \%$ power to detect a proportional difference as small as $\sim 0.12$ between control and Pten KO groups by two-tailed $t$ test at a significance level of 0.01 , which is $>10$-fold more discrete a change than the 1.363-fold increase in spine maturations in Pten KO neurons we observed (i.e., Fig. 8E). Thus, these data suggest that Pten KO neurons have an equivalent ability to stabilize synaptogenic contacts but 


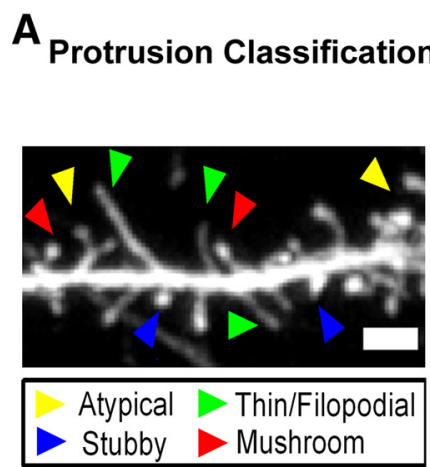

D

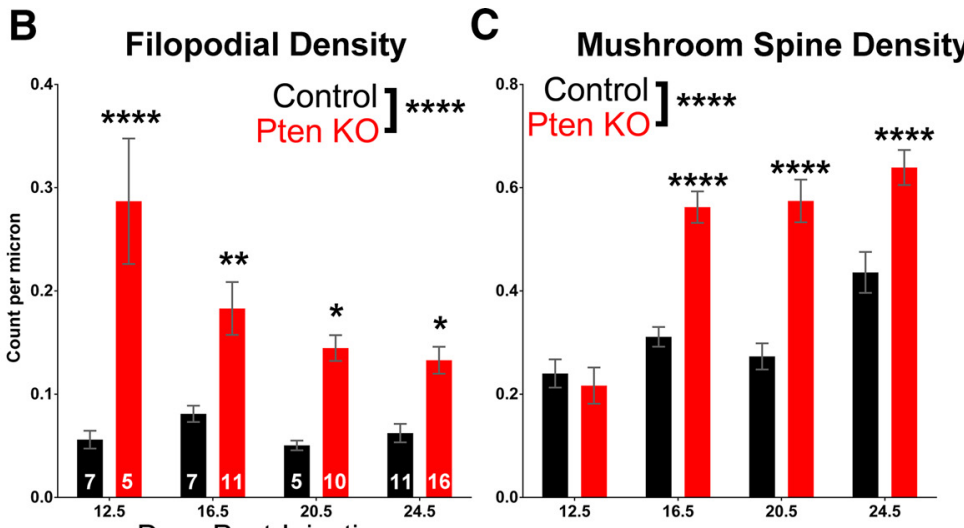

Days Post-Injection

Spine Maturation

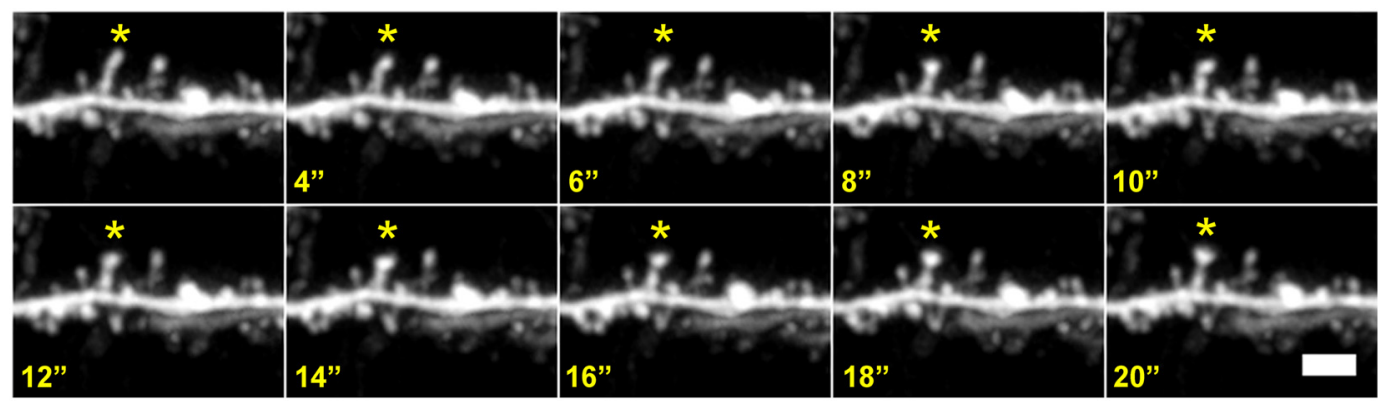

E
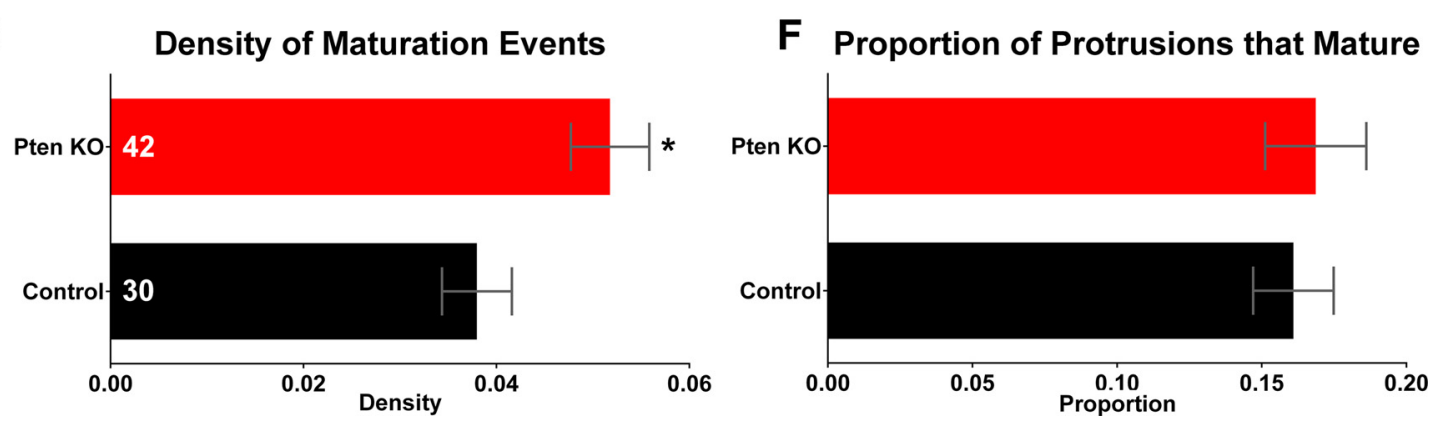

Figure 8. An increased density of dendritic filopodia precedes increased density of mushroom spines in developing Pten $K 0$ neurons. $A$, An exemplary multiphoton image of a live dendritic segment (12.5 DPI, Pten K0) is overlaid with selected protrusions labeled according to manually classified type. Scale bar, $5 \mu \mathrm{m}$. $\boldsymbol{B}$, With age, thin/filopodial protrusion density decreased ( $p=$ $0.0027)$, but in Pten KO cells, overall thin/filopodial protrusion density was increased $(p<0.0001)$; this effect was significant at each DPI. $C$, With age, mushroom spine density increased ( $p$ $<0.0001)$, but in Pten K0 cells, mushroom spine density was further elevated $(p<0.0001)$; this effect was significant at DPI $>12.5 . D,{ }^{*} A$ thin/filopodial protrusion upon a dendritic segment of a $20.5 \mathrm{DPI}$ control neuron undergoes a morphological transition to a mushroom-type spine over the 20 min imaging session. Scale bar, $5 \mu \mathrm{m}$. $\boldsymbol{E}$, The total spatial density of maturation events (as in D) significantly increased with Pten KO ( $p=0.0182)$. , The overall proportion of protrusions that matured was not affected by Pten KO $(p=0.7303)$. Data are mean \pm SEM; $n=$ neurons (indicated in bar bases). $\boldsymbol{B}, \boldsymbol{C}$, Asterisks next to brackets between control and "Pten KO" indicate overall significance of two-way ANOVA. Asterisks above bars indicate significance by Bonferroni's post-test. $\boldsymbol{E}$, $\boldsymbol{F}$, Asterisks indicate significance by $t$ test. ${ }^{*} p<0.05 .{ }^{* *} p<0.005 .{ }^{* * *} p<0.0001$.

do so more often because they have more filopodia, ultimately leading to more functional excitatory synapses.

Accounting for elevated excitatory synaptic input to Pten KO neurons

We sought to test whether the morphological changes we observed in Pten KO neurons not only accompanied, but could also explain, the increased excitatory drive they received. By 20.5-24.5 DPI, the eEPSCs in Pten KO neurons were $4.5 \pm 1.1$-fold that of controls (graphed as eEPSC measured, Fig. 9A; data: Fig. $6 \mathrm{~J}$ ). The increased aggregate eEPSCs could be due to increased dendrite length, spine density, and/or greater synaptic currents at each synapse. We therefore quantified the contribution of each of these changes. Using data from Figure $4 D$, Pten $\mathrm{KO}$ neurons had $1.9 \pm 0.1$-fold more dendritic length versus controls (graphed as total arbor length, Fig. 9A). Using data from the 20.5-24.5 DPI range of Figure $8 C$, Pten $\mathrm{KO}$ neurons had $1.6 \pm 0.07$-fold more mushroom spines than did controls (mushroom spine density, Fig. 9A). Last, using data from Figure $6 K$, aEPSC amplitude (approximating amplitude of quantal events) was $1.3 \pm 0.1$-fold more in Pten KO neurons (aEPSC amplitude, Fig. 9A). We used aEPSC data, rather than mEPSC data, in our models because, although essentially identical in this study, some evidence suggests postsynaptic responses to vesicles undergoing spontaneous versus evoked released differ (Bekkers and Clements, 1999). Furthermore, the evoked events may be more directly related to the increased glutamatergic input. Individually, none of these factors alone was sufficient to explain the enhanced synaptic drive that Pten KO neurons received. However, the product of the increases in arbor length, mushroom spine density, and aEPSC amplitude did yield the predicted enhancement of the eEPSC in Pten KO neurons: $4.1 \pm 0.4$-fold greater (eEPSC predicted, Fig. 9A). This 

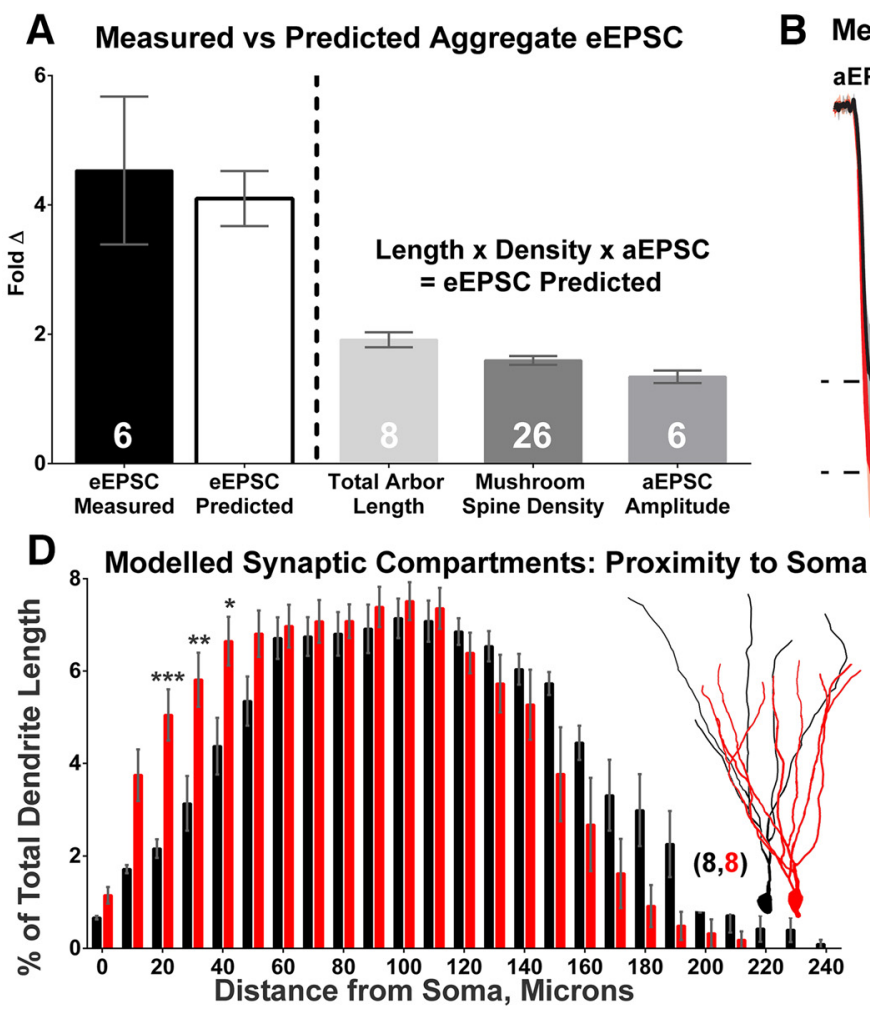

B Measured vs Modelled aEPSC

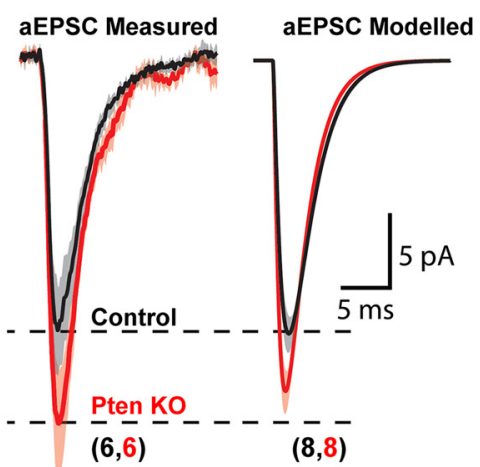

C Peak aEPSC Amplitude

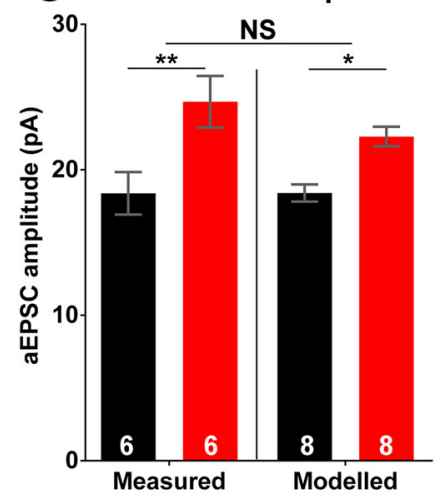

E Components of increased eEPSC in Pten KO
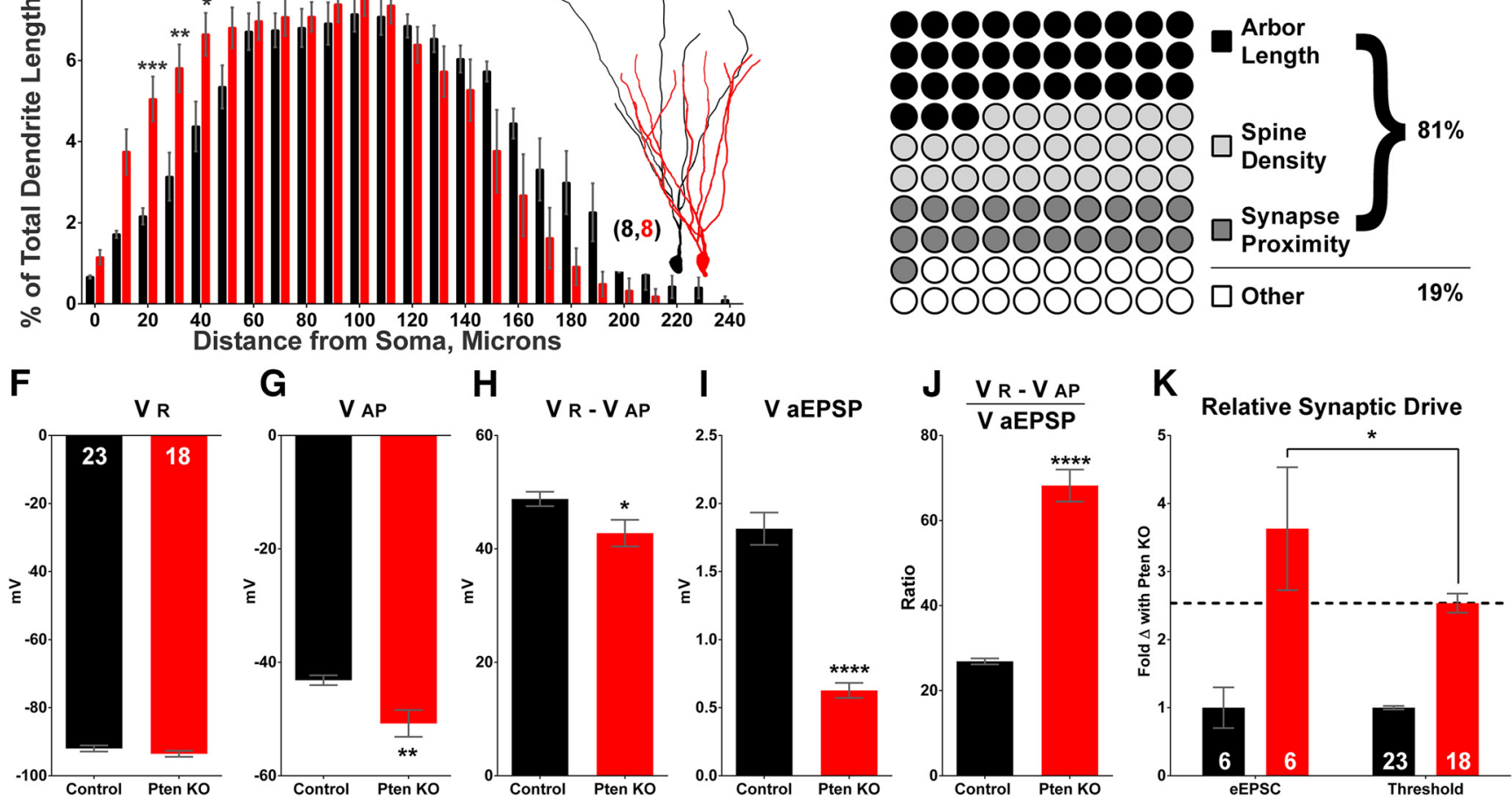

Figure 9. Changes in morphology and excitability explain the increased activity of Pten KO neurons. $\boldsymbol{A}$, Increased eEPSC amplitude from Pten KO (4.532 \pm 1.144 -fold, $\boldsymbol{J})$ is accounted for by Pten KO-associated increases in dendrite length, spine density, and aEPSC amplitude (4.099 \pm 0.425 -fold). $\boldsymbol{B}$, Experimentally measured aEPSCs (Fig. $6 \mathrm{G}$ ) and aEPSCs from simulated neurons based on experimentally determined morphology had similar amplitudes and kinetics. C, Both modeled ( $p=0.027)$ and experimentally recorded $(p=0.002)$ aEPSC amplitudes were increased by Pten K0, with no difference in measured versus modeled approaches (not significant, $p=0.297$ ). $\boldsymbol{D}$, Dendritic compartments were more proximal to the soma in Pten KO neurons. Inset, Reconstructions at 24.5 DPI. $\boldsymbol{E}$, Increased arbor length, spine density, and synapse proximity account for $>80 \%$ of the increased eEPSC in Pten K0 neurons. $\boldsymbol{F}$, Resting potential did not differ with Pten K0. G, Spike threshold was lower with Pten KO ( $p=0.0064)$. $\boldsymbol{H}$, The depolarization to bring neurons to threshold was decreased by Pten $\mathrm{KO}(p=0.0325)$. $\boldsymbol{I}$, aEPSP voltages were smaller with Pten K0 ( $p$ $<0.0001)$.J, It took more aEPSPs (i.e., synaptic inputs) to make a Pten KO neuron fire $(p<0.0001) . K$, In the Pten KO condition, the increase in evoked synaptic inputs was greater than the increase in synaptic inputs required to depolarize Pten KO neurons. Thus, Pten KO neurons are predicted to be more likely to fire in response to afferent stimulation $(p=0.0143)$. Data are mean $\pm S E M ; n=$ neurons (indicated in bar bases). $\boldsymbol{B}$, Data are \pm 2 SE. $\boldsymbol{C}, \boldsymbol{K}$, Asterisks next to brackets between control and "Pten K0" indicate overall significance of two-way ANOVA. Asterisks above bars indicate significance by Bonferroni's post-test. $\boldsymbol{F}-J$, Asterisks indicate significance by $t$ test. ${ }^{*} p<0.05 .{ }^{* *} p<0.005 .{ }^{* * * *} p<0.0001$. NS, Not significant.

predicted elevation of excitatory input was within the experimentally measured range (eEPSC measured, Fig. 9A). Therefore, these data suggest that both the altered dendrite morphology (length and density) and the increased aEPSC amplitude explained the enhanced excitatory drive to Pten KO cells.

We investigated whether the increased aEPSC amplitude in Pten KO cells may also have a morphological basis. We first reconstructed the morphologies of 8 control and 8 Pten KO neurons (i.e., data from Fig. $4 C, D$ ) as models in the NEURON simulation environment (Hines, 1989). For each neuron subtype, experimentally observed age- and Pten KO-dependent differences in dendritic protrusion density were modeled as reciprocal fold changes in dendritic $\mathrm{C}_{\mathrm{m}}$ and $\mathrm{R}_{\mathrm{m}}$ in dendrite compartments. Membrane resistivity $\left(\mathrm{R}_{\mathrm{m}}\right)$ was adjusted so mean input resistance $\left(\mathrm{R}_{\mathrm{N}}\right)$ of the group matched the experimentally determined $\mathrm{R}_{\mathrm{N}}$ of that population. Simulated AMPA-like synaptic conductances (360 pS) were positioned at $1 \mu \mathrm{m}$ intervals along dendrites and independently activated while recording the resultant somatic aEPSCs using simulated single-electrode somatic voltage-clamp $(-93 \mathrm{mV})$. When identical synaptic conductances were generated in the simulated neurons, mean somatic aEPSC amplitudes were larger in Pten KO than in control cells (Fig. 9B; $p=0.027$ ), and we found no difference between simulated and electrophysiological approaches (Fig. 9C; $p=0.297$; two-way ANOVA; 6 
control and $6 \mathrm{KO}$ neurons measured, 8 modeled control and 8 KO neurons). Thus, our simulations could accurately predict the increased aEPSC amplitude found in Pten KO cells, and we could therefore investigate a potential morphological basis for this phenomenon.

Why were the somatic EPSCs, evoked by identical synaptic conductances, larger in Pten KO neurons even though they had lower specific membrane resistivity and had greater dendritic membrane capacitance and conductance? Because somatic capacitance has limited influence on synaptic responses recorded under somatic voltage clamp, the hypertrophy of Pten KO neuron somata was not a factor. However, as reported in Figure $4 D$, Pten KO neurons had more primary dendrites; therefore, a higher proportion of all simulated Pten KO synaptic compartments (i.e., dendritic locations at $1 \mu \mathrm{m}$ intervals) were physically closer to the soma than they were in control cells (Fig. 9D; $1.2 \pm$ 0.04-fold closer, $p<0.0001$; two-way ANOVA; 8 control, $8 \mathrm{KO}$ neurons). That is, aEPSCs were larger in Pten KO cells because synapses were located, on average, more proximal to the soma. By updating our predicted enchantment of the EPSC in Pten KO cells with this information (i.e., as in Fig. 9A), we determined that the relative contributions of these morphological changes (increased dendritic arbor length, mushroom spine density, and synapse proximity to soma) were sufficient to account for the majority $(81 \%)$ of the enhanced excitatory synaptic responses observed in Pten KO neurons (Fig. 9E).

Collectively, we found that Pten $\mathrm{KO}$ neurons were more active in vivo, had altered intrinsic properties, and had morphological properties that accounted for their increased synaptic responsiveness. We therefore tested whether the altered morphological properties and electrophysiological changes induced by Pten $\mathrm{KO}$ could explain their increased firing. Using electrophysiological data from 20.5-24.5 DPI time points (Fig. 2), we found no difference in resting membrane potentials of control versus Pten KO neurons (Fig. 9F; $\mathrm{V}_{\mathrm{R}} ; p=0.229, t$ test; 23 control, $18 \mathrm{KO}$ neurons). However, the membrane potential of Pten KO neurons was more hyperpolarized at action potential threshold (Fig. 9G; $\mathrm{V}_{\mathrm{AP}} ; p=0.0064, t$ test; 23 control, $18 \mathrm{KO}$ neurons). Thus, it took less somatic voltage change to induce action potentials in Pten KO neurons (Fig. $9 H ; \mathrm{V}_{\mathrm{R}}-\mathrm{V}_{\mathrm{AP}} ; p=0.0325, t$ test; 23 control, 18 KO neurons). However, although less depolarization was needed to induce action potentials in Pten $\mathrm{KO}$ neurons, their morphological properties (i.e., much greater somatic capacitance) caused each synaptic conductance to produce a much smaller somatic depolarization (aEPSP). Indeed, mean simulated aEPSP amplitudes were $3 \times$ lower in Pten $\mathrm{KO}$ neurons (Fig. 9I; $\mathrm{V}_{\mathrm{aEPSP}} ; p$ $<0.0001, t$ test; $n=8$ control, $8 \mathrm{KO}$ neurons). We therefore quantified the number of synaptic inputs that would be required to bring control versus Pten KO neurons to threshold from rest (assuming linear integration) (Krueppel et al., 2011) by dividing the difference between resting potential and spike threshold by the aEPSP amplitude. We determined that it would take more synaptic inputs to depolarize a Pten $\mathrm{KO}$ neuron from rest to threshold: for controls, $\sim 27$ synaptic inputs would be necessary to reach threshold, whereas $\sim 68$ inputs would be required for Pten KO neurons (a 2.54-fold increase; Fig. 9J; $\left(\mathrm{V}_{\mathrm{R}}-\mathrm{V}_{\mathrm{AP}}\right) / \mathrm{V}_{\mathrm{aEPSP}}$; $p<0.0001, t$ test; 23 control, $18 \mathrm{KO}$ neurons). Thus, action potential generation in Pten $\mathrm{KO}$ neurons requires the activation of more synapses than does action potential generation in control neurons.

We next determined whether the measured increase in the number of quantal events per evoked current would be sufficient to increase the firing of Pten $\mathrm{KO}$ neurons. We had found that there was a 3.63-fold increase in quantal-like aEPSCs per eEPSC in Pten $\mathrm{KO}$ cells (i.e., data from Fig. $6 \mathrm{~L}$, illustrated in Fig. $9 \mathrm{~K}$, aEPSC). This 3.63-fold increase in events per presynaptic stimulus was greater than the 2.54-fold increase in depolarizing events that our simulations predicted would be necessary for Pten KO cells to reach threshold (i.e., data from Fig. 9J, illustrated in Fig. $9 K$, threshold). The increase in evoked synaptic input was sufficient to overcome the increased number of synaptic events necessary to reach threshold (Fig. $9 K$; $p=0.0143$; two-way ANOVA; $6 \mathrm{KO}$ neurons: events per EPSC, $18 \mathrm{KO}$ neurons: events to threshold). Thus, despite being hypertrophic, Pten KO neurons are more synaptically excitable than controls because of their increased dendritic territory, higher spine density, increased synapse proximity, and more hyperpolarized firing threshold.

\section{Discussion}

The phenotype from Pten KO varies depending on when Pten loss occurs. Constitutive Pten KO is lethal at E7.5 (Di Cristofano et al., 1998). Conditional Pten KO before neuronal differentiation (GFAP-Cre) causes neuronal hypertrophy and seizures in 7-week-old animals, with decreased survival rates after 14 weeks (Backman et al., 2001; Kwon et al., 2001). Inducible Pten KO in postnatal granule neuron progenitors (Gli1-CreER ${ }^{\mathrm{T} 2}$ ) causes seizures, neuronal hypertrophy, and increased dendritic arborization and spine density within 6 weeks (Pun et al., 2012). Pten KO soon after neuronal differentiation (Nse-Cre) also increases both dendritic arborization and spine density and produces seizures (Kwon et al., 2006). However, these changes develop after 8 months (Kwon et al., 2006). In contrast, Pten KO in mature neurons (CaMKII-Cre) does not cause hypertrophy of cell soma or increases in dendritic spine density and only subtly increases dendritic arborization without noted seizure generation (Chow et al., 2009; Sperow et al., 2012). Thus, the severity of somatic hypertrophy, increased spine density, and increased seizure susceptibility varies with the stage at which Pten is deleted. Perhaps because of these varied strategies, there has not been consensus on the mechanisms through which Pten loss alters neuronal physiology.

One consistent finding from previous research and this study is that Pten deletion from developing neurons alters both morphology and physiology. Isolated Pten $\mathrm{KO}$ neurons on glial micro-islands display somatic hypertrophy, dendrite overgrowth, and increased evoked EPSCs and mEPSCs (Weston et al., 2014). Pten KO in cortical neurons of young animals increases dendritic spine density, total dendritic length, and responsiveness to distant afferent neurons (Xiong et al., 2012). Furthermore, deletion of Pten from postnatally generated neurons in developing animals produces somatic hypertrophy, increased spine density, and increased net activity, as evidenced by the emergence of seizures (Pun et al., 2012). These findings demonstrate that loss of Pten during neuronal development causes morphological abnormalities and increased excitability. However, these previous studies did not completely address the mechanistic interrelation of these phenomena. In the present study, retroviral gene deletion coupled to precise electrophysiological and morphological measurements allowed us to study the progression of the Pten $\mathrm{KO}$ phenotype. We found that there is a tight structure-function relationship in these neurons and that the hyperexcitability of Pten KO neurons is chiefly explained by increased recruitment of excitatory synaptic inputs.

The hyperactivity of hypertrophic Pten KO neurons has been a paradox because large neurons need more current to reach threshold and would therefore be predicted to be less excitable. 
However, our data showing increased expression of activity markers in Pten KO cells, Pten KO mice developing seizures (Backman et al., 2001; Kwon et al., 2001; Ogawa et al., 2007; Ljungberg et al., 2009; Pun et al., 2012), and epilepsy in patients with PTEN mutations (Conti et al., 2012; Marchese et al., 2014), all strongly suggest that Pten $\mathrm{KO}$ neurons can be more active in vivo. While we found that Pten $\mathrm{KO}$ neurons required far more intracellular current injection to fire, they fired at lower intensities of afferent stimulation, indicating an increase in depolarizing synaptic drive upon Pten-depleted cells. Our voltage-clamp recordings demonstrated that the increased drive was due to an increase in the number and size of glutamatergic currents. Further, we show that the Pten KO neurons fire at decreased thresholds, have increased action potential amplitudes, decreased afterhyperpolarization, and fire at higher peak frequencies. Thus, we find intrinsic changes that contribute to the hyperactivity of Pten KO neurons as well as large increases in excitatory drive that overcome the increased current necessary to depolarize the larger neurons to firing threshold.

We found that increased synaptic depolarization of Pten KO neurons is due primarily to the increased number of excitatory synaptic sites. An imbalance of excitation to inhibition, as observed here, has been proposed to be a central etiology in neurodevelopmental disorders; and indeed, in animal models, direct alteration of this balance can produce symptoms reminiscent of ASD (Rubenstein and Merzenich, 2003; Yizhar et al., 2011). Mechanistically, we found that Pten KO increased dendritic filopodia density before the increased "mature" mushroom spine density. This finding supports the interpretation that filopodia participate in the formation of excitatory synapses (Fiala et al., 1998; Wierenga et al., 2008). Pten opposes trophic factors that otherwise signal through PI3K activation to increase neuronal filopodial motility and synaptogenesis (Luikart et al., 2008). Further, the phosphatidylinositol 3,4,5-trisphosphate that Pten degrades is critical for the accumulation and maintenance of postsynaptic density proteins and glutamate receptors at nascent spines (Arendt et al., 2010). Thus, the excess excitatory synaptogenesis described here by Pten KO may result from disinhibited synapse formation and/or increased stability of nascent synapses. This idea is supported by studies showing that Pten is an effector for some chemo-repellants (Chadborn et al., 2006; Oinuma et al., 2010; Perdigoto et al., 2011; Henle et al., 2013). An important question for future research is whether the excess synaptic contacts that Pten KO cells make represent an increased number of interactions with appropriate partners or interactions between neurons that would not normally be connected.

Almost all of the increased evoked excitatory current necessary for Pten KO neurons to fire could be accounted for by differences in dendritic architecture. Indeed, the increased total dendritic length, increased dendritic spine density, and increased amplitude of quantal-like aEPSCs (as for mEPSCs) accounted for the increased current necessary to initiate action potentials in Pten KO cells. Furthermore, modeling indicated that the increased aEPSC amplitude was also largely a function of dendritic architecture; the greater number of primary dendrites arising from neuronal soma effectively placed more synapses proximal to the soma in Pten KO cells. However, the amplitude of the recorded quantal events was $10.7 \%$ larger than that predicted by the model. Although this was within the calculated error of our measurements, it could also reflect a molecular change that would increase AMPA currents (i.e., more synaptic AMPA receptors). Additionally, we found a decrease in the voltage threshold of Pten KO neurons, which contributed to their increased excit- ability. In future studies, it could be investigated whether this is due to changes in the Pten-regulated expression or function of voltage-gated ion channels. Our data do not address the relationship of models of plasticity, such as LTP and LTD to morphological changes in Pten neurons (Wang et al., 2006; Fraser et al., 2008; Sperow et al., 2012; Takeuchi et al., 2013). However, our data do demonstrate that the basic physiological and morphological changes elicited by Pten $\mathrm{KO}$ are tightly coupled and are mechanistically involved in generating the increased excitability.

\section{References}

Amiri A, Cho W, Zhou J, Birnbaum SG, Sinton CM, McKay RM, Parada LF (2012) Pten deletion in adult hippocampal neural stem/progenitor cells causes cellular abnormalities and alters neurogenesis. J Neurosci 32: 5880-5890. CrossRef Medline

Arellano JI, Benavides-Piccione R, Defelipe J, Yuste R (2007) Ultrastructure of dendritic spines: correlation between synaptic and spine morphologies. Front Neurosci 1:131-143. CrossRef Medline

Arendt KL, Royo M, Fernández-Monreal M, Knafo S, Petrok CN, Martens JR, Esteban JA (2010) PIP3 controls synaptic function by maintaining AMPA receptor clustering at the postsynaptic membrane. Nat Neurosci 13:36-44. CrossRef Medline

Backman SA, Stambolic V, Suzuki A, Haight J, Elia A, Pretorius J, Tsao MS, Shannon P, Bolon B, Ivy GO, Mak TW (2001) Deletion of Pten in mouse brain causes seizures, ataxia and defects in soma size resembling Lhermitte-Duclos disease. Nat Genet 29:396-403. CrossRef Medline

Bayer SA, Altman J (1974) Hippocampal development in the rat: cytogenesis and morphogenesis examined with autoradiography and low-level X-irradiation. J Comp Neurol 158:55-79. CrossRef Medline

Bekkers JM, Clements JD (1999) Quantal amplitude and quantal variance of strontium-induced asynchronous EPSCs in rat dentate granule neurons. J Physiol 516:227-248. CrossRef Medline

Butler MG, Dasouki MJ, Zhou XP, Talebizadeh Z, Brown M, Takahashi TN, Miles JH, Wang CH, Stratton R, Pilarski R, Eng C (2005) Subset of individuals with autism spectrum disorders and extreme macrocephaly associated with germline PTEN tumour suppressor gene mutations. J Med Genet 42:318-321. CrossRef Medline

Buxbaum JD, Cai G, Chaste P, Nygren G, Goldsmith J, Reichert J, Anckarsäter H, Rastam M, Smith CJ, Silverman JM, Hollander E, Leboyer M, Gillberg C, Verloes A, Betancur C (2007) Mutation screening of the PTEN gene in patients with autism spectrum disorders and macrocephaly. Am J Med Genet 144B:484-491. CrossRef Medline

Chadborn NH, Ahmed AI, Holt MR, Prinjha R, Dunn GA, Jones GE, Eickholt BJ (2006) PTEN couples Sema3A signalling to growth cone collapse. J Cell Sci 119:951-957. CrossRef Medline

Chen TW, Wardill TJ, Sun Y, Pulver SR, Renninger SL, Baohan A, Schreiter ER, Kerr RA, Orger MB, Jayaraman V, Looger LL, Svoboda K, Kim DS (2013) Ultrasensitive fluorescent proteins for imaging neuronal activity. Nature 499:295-300. CrossRef Medline

Chow DK, Groszer M, Pribadi M, Machniki M, Carmichael ST, Liu X, Trachtenberg JT (2009) Laminar and compartmental regulation of dendritic growth in mature cortex. Nat Neurosci 12:116-118. CrossRef Medline

Conti S, Condò M, Posar A, Mari F, Resta N, Renieri A, Neri I, Patrizi A, Parmeggiani A (2012) Phosphatase and tensin homolog (PTEN) gene mutations and autism: literature review and a case report of a patient with Cowden syndrome, autistic disorder, and epilepsy. J Child Neurol 27: 392-397. CrossRef Medline

Di Cristofano A, Pesce B, Cordon-Cardo C, Pandolfi PP (1998) Pten is essential for embryonic development and tumour suppression. Nat Genet 19:348-355. CrossRef Medline

Donnelly ML, Hughes LE, Luke G, Mendoza H, ten Dam E, Gani D, Ryan MD (2001) The 'cleavage' activities of foot-and-mouth disease virus $2 \mathrm{~A}$ sitedirected mutants and naturally occurring ' $2 \mathrm{~A}$-like' sequences. J Gen Virol 82:1027-1041. Medline

Douglas RM, Dragunow M, Robertson HA (1988) High-frequency discharge of dentate granule cells, but not long-term potentiation, induces c-fos protein. Brain Res 464:259-262. CrossRef Medline

Fiala JC, Feinberg M, Popov V, Harris KM (1998) Synaptogenesis via den- 
dritic filopodia in developing hippocampal area CA1. J Neurosci 18: 8900-8911. Medline

Fraser MM, Bayazitov IT, Zakharenko SS, Baker SJ (2008) Phosphatase and tensin homolog, deleted on chromosome 10 deficiency in brain causes defects in synaptic structure, transmission and plasticity, and myelination abnormalities. Neuroscience 151:476-488. CrossRef Medline

Fricano CJ, Despenza T Jr, Frazel PW, Li M, O’Malley AJ, Westbrook GL, Luikart BW (2014) Fatty acids increase neuronal hypertrophy of Pten knockdown neurons. Front Mol Neurosci 7:30. CrossRef Medline

Goffin A, Hoefsloot LH, Bosgoed E, Swillen A, Fryns JP (2001) PTEN mutation in a family with Cowden syndrome and autism. Am J Med Genet 105:521-524. CrossRef Medline

Henle SJ, Carlstrom LP, Cheever TR, Henley JR (2013) Differential role of PTEN phosphatase in chemotactic growth cone guidance. J Biol Chem 288:20837-20842. CrossRef Medline

Hines M (1989) A program for simulation of nerve equations with branching geometries. Int J Biomed Comput 24:55-68. CrossRef Medline

Hobert JA, Embacher R, Mester JL, Frazier TW 2nd, Eng C (2014) Biochemical screening and PTEN mutation analysis in individuals with autism spectrum disorders and macrocephaly. Eur J Hum Genet 22:273-276. CrossRef Medline

Klein S, Sharifi-Hannauer P, Martinez-Agosto JA (2013) Macrocephaly as a clinical indicator of genetic subtypes in autism. Autism Res 6:51-56. CrossRef Medline

Knight ZA, Tan K, Birsoy K, Schmidt S, Garrison JL, Wysocki RW, Emiliano A, Ekstrand MI, Friedman JM (2012) Molecular profiling of activated neurons by phosphorylated ribosome capture. Cell 151:1126-1137. CrossRef Medline

Krueppel R, Remy S, Beck H (2011) Dendritic integration in hippocampal dentate granule cells. Neuron 71:512-528. CrossRef Medline

Kwon CH, Zhu X, Zhang J, Knoop LL, Tharp R, Smeyne RJ, Eberhart CG, Burger PC, Baker SJ (2001) Pten regulates neuronal soma size: a mouse model of Lhermitte-Duclos disease. Nat Genet 29:404-411. CrossRef Medline

Kwon CH, Luikart BW, Powell CM, Zhou J, Matheny SA, Zhang W, Li Y, Baker SJ, Parada LF (2006) Pten regulates neuronal arborization and social interaction in mice. Neuron 50:377-388. CrossRef Medline

Lesche R, Groszer M, Gao J, Wang Y, Messing A, Sun H, Liu X, Wu H (2002) Cre/loxP-mediated inactivation of the murine Pten tumor suppressor gene. Genesis 32:148-149. CrossRef Medline

Ljungberg MC, Sunnen CN, Lugo JN, Anderson AE, D’Arcangelo G (2009) Rapamycin suppresses seizures and neuronal hypertrophy in a mouse model of cortical dysplasia. Dis Model Mech 2:389-398. CrossRef Medline

Lugo JN, Smith GD, Arbuckle EP, White J, Holley AJ, Floruta CM, Ahmed N, Gomez MC, Okonkwo O (2014) Deletion of PTEN produces autismlike behavioral deficits and alterations in synaptic proteins. Front Mol Neurosci 7:27. CrossRef Medline

Luikart BW, Zhang W, Wayman GA, Kwon CH, Westbrook GL, Parada LF (2008) Neurotrophin-dependent dendritic filopodial motility: a convergence on PI3K signaling. J Neurosci 28:7006-7012. CrossRef Medline

Luikart BW, Schnell E, Washburn EK, Bensen AL, Tovar KR, Westbrook GL (2011a) Pten knockdown in vivo increases excitatory drive onto dentate granule cells. J Neurosci 31:4345-4354. CrossRef Medline

Luikart BW, Bensen AL, Washburn EK, Perederiy JV, Su KG, Li Y, Kernie SG, Parada LF, Westbrook GL (2011b) miR-132 mediates the integration of newborn neurons into the adult dentate gyrus. PLoS One 6:e19077. CrossRef Medline

Marchese M, Conti V, Valvo G, Moro F, Muratori F, Tancredi R, Santorelli FM, Guerrini R, Sicca F (2014) Autism-epilepsy phenotype with macrocephaly suggests PTEN, but not GLIALCAM, genetic screening. BMC Med Genet 15:26. CrossRef Medline

McBride KL, Varga EA, Pastore MT, Prior TW, Manickam K, Atkin JF, Herman GE (2010) Confirmation study of PTEN mutations among individuals with autism or developmental delays/mental retardation and macrocephaly. Autism Res 3:137-141. CrossRef Medline

Ogawa S, Kwon CH, Zhou J, Koovakkattu D, Parada LF, Sinton CM (2007)
A seizure-prone phenotype is associated with altered free-running rhythm in Pten mutant mice. Brain Res 1168:112-123. CrossRef Medline

Oinuma I, Ito Y, Katoh H, Negishi M (2010) Semaphorin 4D/Plexin-B1 stimulates PTEN activity through R-Ras GTPase-activating protein activity, inducing growth cone collapse in hippocampal neurons. J Biol Chem 285:28200-28209. CrossRef Medline

Orrico A, Galli L, Buoni S, Orsi A, Vonella G, Sorrentino V (2009) Novel PTEN mutations in neurodevelopmental disorders and macrocephaly. Clin Genet 75:195-198. CrossRef Medline

Perdigoto AL, Chaudhry N, Barnes GN, Filbin MT, Carter BD (2011) A novel role for PTEN in the inhibition of neurite outgrowth by myelinassociated glycoprotein in cortical neurons. Mol Cell Neurosci 46:235244. CrossRef Medline

Peters A, Kaiserman-Abramof IR (1970) The small pyramidal neuron of the rat cerebral cortex: the perikaryon, dendrites and spines. J Anat 127:321355. CrossRef Medline

Pun RY, Rolle IJ, Lasarge CL, Hosford BE, Rosen JM, Uhl JD, Schmeltzer SN, Faulkner C, Bronson SL, Murphy BL, Richards DA, Holland KD, Danzer SC (2012) Excessive activation of mTOR in postnatally generated granule cells is sufficient to cause epilepsy. Neuron 75:1022-1034. CrossRef Medline

Rubenstein JL, Merzenich MM (2003) Model of autism: increased ratio of excitation/inhibition in key neural systems. Genes Brain Behav 2:255267. CrossRef Medline

Rudolph S, Overstreet-Wadiche L, Wadiche JI (2011) Desynchronization of multivesicular release enhances Purkinje cell output. Neuron 70:9911004. CrossRef Medline

Sperow M, Berry RB, Bayazitov IT, Zhu G, Baker SJ, Zakharenko SS (2012) Phosphatase and tensin homologue (PTEN) regulates synaptic plasticity independently of its effect on neuronal morphology and migration. J Physiol 590:777-792. CrossRef Medline

Stein MT, Elias ER, Saenz M, Pickler L, Reynolds A (2010) Autistic spectrum disorder in a 9-year-old girl with macrocephaly. J Dev Behav Pediatr 31:632-634. CrossRef Medline

Takeuchi K, Gertner MJ, Zhou J, Parada LF, Bennett MV, Zukin RS (2013) Dysregulation of synaptic plasticity precedes appearance of morphological defects in a Pten conditional knock-out mouse model of autism. Proc Natl Acad Sci U S A 110:4738-4743. CrossRef Medline

Tuchman R, Cuccaro M, Alessandri M (2010) Autism and epilepsy: historical perspective. Brain Dev 32:709-718. CrossRef Medline

Varga EA, Pastore M, Prior T, Herman GE, McBride KL (2009) The prevalence of PTEN mutations in a clinical pediatric cohort with autism spectrum disorders, developmental delay, and macrocephaly. Genet Med 11: 111-117. CrossRef Medline

Wang Y, Cheng A, Mattson MP (2006) The PTEN phosphatase is essential for long-term depression of hippocampal synapses. Neuromolecular Med 8:329-336. CrossRef Medline

Weston MC, Chen H, Swann JW (2014) Loss of mTOR repressors Tsc1 or Pten has divergent effects on excitatory and inhibitory synaptic transmission in single hippocampal neuron cultures. Front Mol Neurosci 7:1. CrossRef Medline

Wierenga CJ, Becker N, Bonhoeffer T (2008) GABAergic synapses are formed without the involvement of dendritic protrusions. Nat Neurosci 11:1044-1052. CrossRef Medline

Xiong Q, Oviedo HV, Trotman LC, Zador AM (2012) PTEN regulation of local and long-range connections in mouse auditory cortex. J Neurosci 32:1643-1652. CrossRef Medline

Yizhar O, Fenno LE, Prigge M, Schneider F, Davidson TJ, O'Shea DJ, Sohal VS, Goshen I, Finkelstein J, Paz JT, Stehfest K, Fudim R, Ramakrishnan C, Huguenard JR, Hegemann P, Deisseroth K (2011) Neocortical excitation/inhibition balance in information processing and social dysfunction. Nature 477:171-178. CrossRef Medline

Zhou J, Blundell J, Ogawa S, Kwon CH, Zhang W, Sinton C, Powell CM, Parada LF (2009) Pharmacological inhibition of mTORC1 suppresses anatomical, cellular, and behavioral abnormalities in neural-specific Pten knock-out mice. J Neurosci 29:1773-1783. CrossRef Medline 